\title{
Gamma-Ray Bursts as Sources of Strong Magnetic Fields
}

\author{
Jonathan Granot, Tsvi Piran, Omer Bromberg, \\ Judith L. Racusin, and Frédéric Daigne
}

\begin{abstract}
Gamma-Ray Bursts (GRBs) are the strongest explosions in the Universe, which due to their extreme character likely involve some of the strongest magnetic fields in nature. This review discusses the possible roles of magnetic fields in GRBs, from their central engines, through the launching, acceleration and collimation of their ultra-relativistic jets, to the dissipation and particle acceleration that power their $\gamma$-ray emission, and the powerful blast wave they drive into the surrounding medium that generates their long-lived afterglow emission. An emphasis is put on particular areas in which there have been interesting developments in recent years.
\end{abstract}

Keywords Gamma-ray bursts · Magnetic fields · MHD · Neutron stars · Jets · Radiation mechanisms: non-thermal

Jonathan Granot

Department of Natural Sciences, The Open University of Israel, 1 University Road, P.O. Box 808, Ra'anana 4353701, Israel

E-mail: granot@openu.ac.il

Tsvi Piran

Racah Institute of Physics, The Hebrew University of Jerusalem, Jerusalem 91904, Israel

E-mail: tsvi@phys.huji.ac.il

Omer Bromberg

Department of Astrophysical Sciences, Princeton University, 4 Ivy Ln., Princeton NJ 08544, USA

E-mail: omerb@astro.princeton.edu

Judith L. Racusin

Astrophysics Science Division, NASA Goddard Space Flight Center, Greenbelt MD, USA

E-mail: judith.racusin@nasa.gov

Frédéric Daigne

Institut d'Astrophysique de Paris, UMR 7095 Université Pierre et Marie Curie; CNRS, 98 bis, boulevard Arago, 75014 Paris, France

E-mail: daigne@iap.fr 


\section{Introduction}

Gamma-ray Bursts (GRBs) are among the most extreme objects in the Universe. They are the most luminous cosmic explosions, and therefore serve as beacons at the edge of the visible Universe that can be used as cosmic probes. GRBs provide short timescale insight into end-stage stellar evolution, and serve as probes of extreme physics such as strong gravity, very large densities and magnetic fields, extremely energetic particles, and relativistic bulk motions. They are also promising sources of high-energy neutrinos and gravitational waves.

GRBs can be roughly divided into two main sub-classes: (i) Long-duration ( $\gtrsim 2 \mathrm{~s})$ soft-spectrum bursts that are found in star-forming regions and are associated with broad-lined Type Ic supernovae, implying a massive star progenitor, which is most likely low-metalicity and rapidly rotating near this cataclysmic end of its life, and lives in a gas-rich environment not far from where it was formed. In order to produce a GRB, the central engine must drive a strong relativistic jet that bores its way through the stellar envelope and produces the GRB well outside of the progenitor star; (ii) Short-duration $(\lesssim 2 \mathrm{~s})$ hard-spectrum bursts that are thought to arise from the merger of a binary neutron star system (or a neutron star and a stellar-mass black hole) that emits gravitational waves as it inspirals and coalesces, producing a central engine driven jet. Such systems live in low density environments, possibly with a prior supernova kick that pushed them into the outskirts of their host galaxies. A third sub-class, whose importance was realized only recently (Soderberg et al. 2006 Campana et al. 2006, Liang et al. 2007, Virgili et al. 2009, Bromberg et al. 2011. 2012; Nakar and Sari 2012), involves low-luminosity GRBs, whose overall isotropic equivalent radiated energy is $E_{\gamma, \text { iso }} \lesssim 10^{49} \mathrm{erg}$. They also typically have a smooth, single-peaked lightcurve, and their $v F_{v}$ spectrum typically peaks at a lower than average photon energy (usually $E_{\mathrm{p}} \lesssim 100 \mathrm{keV}$ ). While observed rarely, because of their low luminosity, they are the most numerous group in nature (in terms of their rate per unit volume). They most likely do not arise from the same emission mechanism as regular long GRBs (e.g., Bromberg et al.|2012, Nakar and Sari 2012).

The phenomenology of GRBs is generally separated into two observational phases: the prompt emission and the afterglow. These two phases are traditionally differentiated largely based upon instrumental measurement methods, but they do seem to also be physically distinct - they arise from different emission mechanisms and occur at different distances from the central source. However, the dividing lines between the prompt emission and the afterglow have blurred in recent years. In the standard Fireball model (e.g., Piran 1999, 2004, Kumar and Zhang 2015), the prompt emission (i.e. the burst of $\gamma$-rays) is due to dissipative internal shocks within the outflow, while the long-lived broadband afterglow is the result of the jet driving a strong relativistic forward shock into the surrounding medium as it decelerates and transfers its energy to the shocked external medium. Unless the outflow is highly magnetized when it is decelerated by the external medium, this deceleration can occur through a strong reverse shock that results in a bright optical flash, which is also sometimes detected (e.g., Akerlof et al. 1999, Sari and Piran 1999; Mundell et al. 2007b; Racusin et al. 2008: Vestrand et al. 2014) just after the onset of the prompt emission, and decays largely independently of the forward shock emission. 
The field of GRBs is relatively young, with several revolutions in our understanding of these objects thanks to new observations over the last two decades. The role of magnetic fields in GRBs is relevant to many topics in this field. They affect the properties of the compact object (neutron star and/or black hole) that powers the central engine, and how it launches the jet. Magnetic fields may also play an important role in the acceleration and collimation of the relativistic jets in GRBs, as well as in their composition. They can contribute to the energy dissipation and particle acceleration that powers the prompt GRB emission, and may play a key role in its emission mechanism. A strong magnetic field can suppress the reverse shock and its emission. During the afterglow, the amplification of the weak magnetic field in the external medium by the afterglow shock and its subsequent behavior in the shocked external medium downstream of the shock play a key role in the particle acceleration by the shock and in the shaping of the afterglow emission. In fact, it seems hard to find any important part of GRB physics where magnetic fields might be safely ignored.

In this review, we explore the evidence for extreme magnetic fields in GRBs, and how magnetic fields are intertwined with our understanding of the mechanisms that produce the relativistic jets that power these objects. As we cannot cover here all of the relevant topics in detail, we have instead chosen to focus on specific topics in which there has been recent progress (see e.g. Piran 2005, for an earlier review). First, in $\$ 2$ a brief overview is given on the progenitors of both long and short GRBs, with the main thrust being devoted to the possible role of millisecond magnetars newly born, very rapidly rotating and highly magnetized neutron stars - a topic that has recently received a lot of attention in the literature and in the GRB community. Next, 3 discusses the dynamics of GRB jets. It starts with long GRB jets as they bore their way out of their massive star progenitors, and then moves on to discuss more generally the possible role of magnetic fields in the acceleration and collimation of GRB jets, both in steady-state and highly time-variable outflows, as well as in the interaction of the jet with the external medium and the reverse shock. In $\$ 4$ we discuss the role of magnetic fields in the dissipation and radiation that power the prompt $\gamma$-ray emission, and what GRB observations can tell us about the conditions within the emitting region. Finally, $\$ 5$ is devoted to the role of magnetic fields in the afterglow. It focuses on their effects on the afterglow and reverse shock emission and their polarization, and how this can teach us about the magnetic field structure in the GRB outflow and its amplification in the afterglow shock as well as its structure and possible decay further downstream of this shock. Our conclusions are discussed in \$6.

\section{GRB Progenitors, Central Engine, and the Role of Magnetars}

Long duration GRBs are associated with Type Ic supernovae (e.g., Woosley and Bloom 2006), which directly relate them to the death of massive stars stripped of their hydrogen and helium. This supports the popular Collapsar model (Woosley 1993, MacFadyen and Woosley 1999) in which a central engine lunches a relativistic jet that penetrates the stellar envelope and powers the GRB. Typically, within the Collapsar models the central engine is considered to be a an accreting newly-formed 
stellar-mass black hole at the center of the progenitor star. The most popular model for short duration GRBs features the merger of two neutron stars in a tight binary system (Eichler et al. 1989), which may again form a black hole surrounded by an accretion disk as they coalesce. Therefore, in both long and short duration GRBs, despite their very different progenitors, the central engine that is formed during the explosion and launches the relativistic jets might still be similar in nature - accretion onto a newly formed black hole.

An attractive alternative possibility that has gained popularity in recent years is that GRB central engines may involve magnetars (Usov|1992; Duncan and Thompson 1992, Bucciantini et al.2008, Dall'Osso et al. 2009) - highly magnetized neutron stars with surface magnetic fields of order $B \sim 10^{15} \mathrm{G}$, which in this case are newly born and very rapidly rotating, with $\sim 1$ ms periods (and hence dubbed millisecond magnetars). In this model the main energy source is the neutron star's rotational energy, and a very strong magnetic field is needed for a rapid extraction of this rotational energy and to channel it into a relativistic outflow. A rapidly rotating neutron star may arise in the collapse of a rotating stellar core and the magnetic field can be amplified in this collapse (e.g., Duncan and Thompson 1992). As the magnetar's energy is naturally extracted in the form of a Poynting flux (though this flux is initially not significantly collimated) it naturally leads to a magnetically dominated outflow. Collimation of the outflow into a narrow jet may, however, be facilitated by the interaction of the outgoing strong magnetohydrodynamic (MHD) wind with the progenitor star's envelope (e.g., Bucciantini et al. 2007, 2008, 2009, Bromberg et al. 2014).

After the first $\sim 10-100 \mathrm{~s}$ or so from the neutron star formation, the neutrinodriven winds subside and the baryon loading on the MHD wind significantly decreases. As a result the initial wind magnetization parameter $\sigma_{0}$ significantly increases and becomes $\gg 1$ (the magnetization parameter $\sigma$ is the Poynting-to-matter energy flux ratio, or proper enthalpy density ratio). At this stage, the neutron star spin-down and its associate luminosity are approximately given by the magnetic dipole in vacuum formula (which also approximately holds in the force-free regime; Spitkovsky 2006),

$$
L(t)=\frac{B^{2} R^{6} \Omega_{0}^{4} /\left(6 c^{3}\right)}{\left[1+2 B^{2} R^{6} \Omega_{0}^{2} t /\left(6 I c^{3}\right)\right]^{2}}=\frac{E_{0}}{t_{0}\left(1+t / t_{0}\right)^{2}} \approx L_{0} \times\left\{\begin{array}{cl}
1 & \left(t<t_{0}\right), \\
\left(t / t_{0}\right)^{-2} & \left(t>t_{0}\right),
\end{array}\right.
$$

where $I$ is the neutron star's moment of inertia, $R$ is its radius, $B$ is the surface dipole magnetic field at the pole, $\Omega_{0}$ is the initial angular velocity, $E_{0} \approx \frac{1}{2} I \Omega_{0}^{2}$ is the initial rotational energy, $L_{0}=E_{0} / t_{0}$ is the initial spin-down luminosity and

$$
t_{0}=\frac{3 I c^{3}}{B^{2} R^{6} \Omega_{0}^{2}} \approx 2 \times 10^{3}\left(\frac{B}{10^{15} \mathrm{G}}\right)^{-2}\left(\frac{P}{1 \mathrm{~ms}}\right)^{2} \mathrm{~s}
$$

is the initial spin-down time (using typical values of $R \approx 10 \mathrm{~km}, I \approx 10^{45} \mathrm{~g} \mathrm{~cm}^{2}$ ). This spindown luminosity initially (at $t<t_{0}$ ) has a plateau at $L_{0}$, and then (at $t>t_{0}$ ) falls off as $t^{-2}$. Both $L_{0}$ and $t_{0}$ can be tuned with the proper choice of the initial angular velocity $\Omega_{0}$ and the magnetic field $B$. With a choice of $B \sim 10^{15.5-16} \mathrm{G}$ one 
can arrange $t_{0}$ fit the prompt duration in which case the magnetar is invoked to power the prompt GRB. With a lower magnetic field of order $10^{14.5-15} \mathrm{G}, t_{0}$ is of order several thousand seconds, comparable to the duration of the plateau phase in some X-ray afterglows.

The magnetar model gained a lot of popularity with the discovery by Swift of plateaus in the X-ray afterglow light curves of many GRBs (Nousek et al. 2006. Zhang et al. 2006), whose shape resembles the overall shape of Magnetar's spindown luminosity (Troja et al. 2007; Dall'Osso et al. 2009, 2011; Rowlinson et al. 2013, 2014). Somewhat surprisingly, even though this tentative evidence ${ }^{1}$ for magnetar-like activity was obtained for the afterglow phase, it was interpreted in the community as evidence for a magnetar operating as the main source of energy for the prompt emission as well. Both interpretations face some difficulties.

The magnetic field needed to produce the prompt emission is larger by about one order of magnitude than the one observed even in the strongest magnetars. This may not be that puzzling as there is ample evidence of magnetic field decay in magnetars (Dall'Osso et al. 2012), and the observed magnetars are typically a few thousand years old. It is possible that the magnetic fields of newborn magnetars are large enough.

A more serious problem concerns the energy budget. The rotational energy of a typical neutron star, even when rotating at breakup velocity, is at best marginally sufficient to power the most powerful GRBs (Cenko et al.2010). This is especially so if we also take into account the efficiency of converting this rotational energy into the prompt flux of $\gamma$-rays. Of course, magnetars could still power less energetic GRBs. However, this would require one to invoke two kinds of central engines, as a different energy source would be needed to power the most energetic GRBs.

Even more perplexing is the situation concerning the longer duration plateaus in the afterglow light curves. Here, the needed values of magnetic fields are indeed typical for those arising in the observed magnetars, and the overall energy budget is reasonable as well. However, another question arises: if a low magnetic field magnetar has powered the afterglow plateau, then what has powered the prompt GRB? Can a magnetar fire twice? The simple answer, according to Eq. (1), is no. This is as long as the magnetic field remains constant during the slowdown time scale. However, one can come up with a fine-tuned model in which the magnetic field decays on a timescale shorter than $t_{0}$. In this case the duration of the magnetar activity is not determined by $t_{0}$, but by the magnetic field decay time. Once the magnetic field has decayed, a second slower magnetar phase appears with a new $t_{0}$. Overall such a model requires a the magnetic field that is extremely large initially, leading to the prompt emission and then it decreases, just at the right time (and before all the rotational energy is exhausted) to a lower level in which the weaker magnetar powers (using the remaining rotational energy) the afterglow plateau.

1 These plateaus have several alternative explanations, which are at least as compelling as the magnetar explanation, such as promptly ejected slow material that gradually catches up with the afterglow shock (Nousek et al. 2006 Granot and Kumar 2006), time-varying afterglow shock microphysical parameters (Granot et al. 2006), viewing angle effects (Eichler and Granot 2006), or a two-component jet (Peng et al. 2005 Granot et al. 2006). 
An alternative option is as follows: in the first $\sim 10-100 \mathrm{~s}$ or so after the formation of the neutron star, the strong neutrino-driven winds cause a large baryon loading on the MHD wind that prevents the formation of a very high initial magnetization $\left(\sigma_{0} \lesssim 10-100\right)$. Therefore, during at least part of this time the spindown luminosity can significantly deviate from the form Eq. (1) - the formula for a magnetic dipole in vacuum - and may in fact be significantly higher, and closer to the result for a magnetic monopole in vacuum, since most of the magnetic field lines are opened by the strong baryon loading (e.g., Metzger et al.|2011). This can increase the spindown luminosity by a factor of $\sim\left(R_{L} / R\right)^{2} \sim 10^{1.5}$, where $R_{L}=c / \Omega_{0}$ is the initial value of the light-cylinder radius. However, as in the early magnetic field decay scenario mentioned above, also this solution would require fine tuning in order to extract just the right amount of rotational energy over just the right timescale. Moreover, in this case the bulk of the large amount of energy that is released on the timescale of the prompt GRB is given to relatively low- $\sigma_{0}$ baryon-rich material, which could not attain sufficiently large asymptotic Lorentz factors that are needed to power a GRB.

Another possible solution to this problem was suggested recently (Rezzolla and Kumar 2015, Ciolfi and Siegel 2015) - the "time reversal model", which postulates magnetar activity for the plateau but an accretion disk for the prompt phase. According to this model, first a magnetar with $t_{0} \sim 10^{4} \mathrm{~s}$ is born and launches a fast MHD wind whose interaction with slower matter that was ejected earlier produces the afterglow plateau. In this scenario the magnetar is a supramassive neutron star, i.e. supported against gravitational collapse by its very fast rotation, so once it spins down significantly it collapses to a black hole, and an accretion disk that forms during this collapse powers the prompt emission ${ }^{2}$ In spite of this reversed time sequence the plateau is observed after the prompt emission because it involved the interaction of the winds and this phase introduces a time delay (in the observer frame). While it is appealing, this model requires the formation of a disk during the collapse of the supramassive neutron star. However, Margalit et al. (2015) have recently argued that this is impossible.

A different possible solution is if the prompt GRB is powered by the energy in a strong initial differential rotation (Kluźniak and Ruderman 1998). The strong differential rotation winds-up strong toroidal magnetic field loops, which are buoyantly pushed out of the neutron star surface and power the prompt GRB. This lasts until they exhaust all of the differential rotation energy on the timescale of the prompt GRB emission of long duration GRBs. The rotational energy of the remaining uniformly rotating neutron star could then power the plateaus on its longer magnetic dipole spindown time $t_{0}$. This might possibly work for long duration GRBs that are not too energetic (as the energy in differential rotation is somewhat lower than the total rotational energy).

Recently, it was suggested that millisecond magnetars might also be at work in short duration GRBs (e.g., Fan and Xu 2006, Rosswog 2007; Metzger et al.|2008 Rowlinson et al. 2010; Bucciantini et al. 2012, Rowlinson et al. 2013; Fan et al. 2013a; Gompertz et al.2014). Newly formed millisecond magnetars were suggested

2 This scenario is rather similar to the "supranova model" that was suggested much earlier Vietri and Stella 1998 1999). 
to produce the extended emission seen on a timescale of $\sim 10^{2} \mathrm{~s}$ in some short GRBs (e.g. Metzger et al.|2008; Bucciantini et al.2012). In this picture the initial short GRB spike may arise from short-lived accretion following the merger. The extended emission is driven by the spindown power that is released over $\sim 10^{2} \mathrm{~s}$, and takes several seconds to break out of the surrounding mildly relativistic material that is ejected quasi-isotropically during the merger. In this scenario, however, the jets that power the short GRB itself (the initial hard spike) are launched within the first second or so after the formation of a newly-born millisecond magnetar, when the neutrino-driven wind is very vigorous, the magnetization is low $\left(\sigma_{0} \lesssim 1\right)$, and the baryon loading is very significant near the star and the inner accretion disc where the jet may be launched. It is therefore unclear whether in this case the jets could eventually reach a high enough Lorentz factor to produce the GRB. Moreover, in a binary merger the neutron star is formed extremely rapidly rotating (near breakup), and its rotational energy of a few $\times 10^{52} \mathrm{erg}$ is eventually injected into the afterglow shock. This should naturally produce a bright afterglow emission while the observed afterglows of short GRBs (either with or without an extended emission) are typically much dimmer than those of long GRBs. While this might in part be attributed to a lower external density on average, this cannot fully account for the dimmer afterglows of short GRBs essentially over the entire broad-band spectrum, from radio to $\mathrm{GeV}$ energies.

\section{Jet Propagation and Dynamics}

The question of the jet composition is still a major issue in our understanding of GRBs. It affects the location of the emission site, the mechanism of the emission and the particle acceleration. There are two main possibilities that are commonly discussed in the literature: a hydrodynamic jet and a Poynting flux dominated jet (for the jet dynamics discussed here we do not make the distinction between baryonic and $e^{ \pm}$ pairs particle content). The main advantage of a hydrodynamic jet is fast and robust acceleration, which allows the jet to reach very high Lorentz factors relatively close to the central source. Magnetic acceleration, on the other hand, is slower and less robust. However, hydromagnetic jet launching implies dynamically strong magnetic fields near the central source, which can naturally avoid excessive baryonic loading into the central part of the jet, and thus allow it to reach large asymptotic Lorentz factors far from the source. The required very small baryon loading is hard to naturally achieve in a purely hydrodynamic jet. Here we focus on the possible role of magnetic fields in the jet dynamics and propagation, while keeping in mind these two main options for the jet composition.

\subsection{Jet Propagation in the Stellar Envelope}

In order to produce a regular GRB, a collapsar jet needs to successfully break out of its progenitor star. After it breaks out, the jet can accelerate freely and eventually generate the observed $\gamma$-ray photons far from the star in a region where they can escape (see \$4). Before it emerges from the stellar surface, the jet propagates inside 
the star by pushing the stellar material in front of it, forming a bow shock ahead of the jet. The stellar material that crosses this shock is heated and forms a cocoon around the jet, which in turn applies pressure on the jet and collimates it. The collimated jet propagates at a different velocity than a freely expanding jet. It continuously injects energy into the cocoon through a slower moving head that forms at the front of the jet. The head dissipates the jet's energy and channels it into the cocoon. Therefore, the continuous propagation of the jet through the star depends on the supply of fresh energy from the source. If the engine stops injecting energy, the head will essentially stop propagating once the information about the energy cutoff will reach it, and the jet will fail. The breakout time, $t_{\mathrm{b}}$, is defined as the time of the engine shutoff for which the information about the shutoff reaches the jet's head when it is at the edge of the star. If the engine stops working at a time $t_{e}<t_{\mathrm{b}}$, the head will "feel" this cutoff while it is inside the star and will stop propagating. In this case the jet will not break out and it will not produce a regular GRB ${ }^{3}$. Since the information travels outwards at very close to the speed of light, the breakout time is related to the time at which the jet's head reaches the outer edge of the star through

$$
t_{\mathrm{b}}=\int_{0}^{R_{*}} \frac{d z}{\beta_{\mathrm{h}}(z) c}-\frac{R_{*}}{c} \equiv \frac{R_{*}}{c} \frac{1-\bar{\beta}_{\mathrm{h}}}{\bar{\beta}_{\mathrm{h}}},
$$

where $\beta_{\mathrm{h}}(z) c$ is the instantaneous velocity of the jet's head at a distance $z$ from the central source (along its symmetry axis, using cylindrical coordinates), and $\bar{\beta}_{\mathrm{h}} c$ is its average velocity.

Simple analytic solutions to Eq. (3) can be obtained in two limits: (i) a nonrelativistic limit, characterized by a proper speed (in units of $c$ ) $u_{\mathrm{h}}=\Gamma_{\mathrm{h}} \beta_{\mathrm{h}} \ll 1$ (where $\Gamma_{\mathrm{h}}$ is the head's Lorentz factor) in which $t_{\mathrm{b}} \simeq R_{*} / \bar{\beta}_{\mathrm{h}} c$, and (ii) the relativistic limit, characterized by $u_{\mathrm{h}} \gg 1$, in which $t_{\mathrm{b}} \simeq R_{*} / 2 \bar{\Gamma}_{\mathrm{h}}^{2} c$. The transition between these two limits occurs when $t_{\mathrm{b}} \simeq R_{*} / c$, which according to Eq. 3 . corresponds to $\bar{\beta}_{\mathrm{h}} \simeq 1 / 2$. The jet's head is initially sub-relativistic, but it accelerates in the steep density profile inside the $\operatorname{star}(\xi=-d \log \rho / d \log r>2)$. Therefore, if the jet becomes relativistic at some radius, $R_{\text {rel }}$, where $u_{\mathrm{h}} \simeq 1$, then it will remain so until it will break out.

In order to calculate the breakout time ones needs a proper model for the propagation of the jet in the star. Such a model needs to consider the evolution of the jet and the cocoon self-consistently, as they affect one another. The propagation velocity of the head is determined by its cross section, which is set by the collimation of the jet. The head's velocity, in turn, controls the energy injection into the cocoon, which determines the collimating pressure. The dynamics of this system can be described in a relatively simple way in two extreme cases of a purely hydrodynamic jet and a purely electromagnetic (Poynting flux dominated) jet.

\subsubsection{The Breakout Time of Collapsar Jets}

Close to the injection point the jet's internal pressure, $p_{j}$, is much larger than the cocoon's pressure, $p_{c}$. Therefore, initially the jet material expands freely until the col-

\footnotetext{
3 A failed jet produces, most likely, a low-luminosity GRB when a shock wave generated by the dissipated energy breaks out from the seller envelope.
} 
limation point where the jet's pressure equals the cocoon's pressure, $p_{\mathrm{j}}=p_{\mathrm{c}}$. Above this point the jet is collimated by the cocoon's pressure, and its behavior depends on its magnetization.

In the hydrodynamic case, the collimation of the jet leads to the formation of a collimation shock at the base of the jet (e.g., Bromberg and Levinson 2007). Above this point the jet maintains a roughly cylindrical shape due to a relatively uniform pressure in the cocoon (e.g., Zhang et al.|2003; Matzner 2003, Bromberg et al. 2011). The jet material remains relativistic with a roughly constant Lorentz factor $\Gamma_{\mathrm{j}} \sim 1 / \theta_{0}$, where $\theta_{0}$ is the jet injection (or initial) half-opening angle. At the head of the jet the relativistic jet material decelerates abruptly through a strong reverse shock. Since the jet is roughly cylindrical upstream of the reverse shock, the width of the head its cylindrical radius $r_{j}$ and its corresponding cross-section $\Sigma_{j}=\pi r_{j}^{2}$, are set by the width and cross-section of the jet at the collimation point, which are shown to be

$$
\Sigma_{j}=\pi r_{j}^{2} \simeq \frac{L_{j} \theta_{0}^{2}}{4 c p_{c}}
$$

where $L_{j}$ is the (one sided) jet luminosity. The velocity of the head was shown (Matzner 2003, Bromberg et al. 2011) to follow

$$
\beta_{h}=\frac{\beta_{j}}{1+\tilde{L}^{-1 / 2}}
$$

where the dimensionless parameter

$$
\tilde{L}=\frac{\rho_{j} h_{j} \Gamma_{j}^{2}}{\rho_{a}} \simeq \frac{L_{j}}{\Sigma_{j} \rho_{a} c^{3}},
$$

represents the ratio between the energy density of the jet $\left(L_{j} / \Sigma_{j} c\right)$ and the rest-mass energy density of the surrounding medium $\left(\rho_{a} c^{2}\right)$ at the location of the head. Here $h_{j}=1+4 p_{j} / \rho_{j} c^{2}$ is the dimensionless specific enthalpy of the jet material just upstream of the termination shock at the base of its head.

Bromberg et al. (2011) have obtained approximate analytic expressions for the propagation velocity of a hydrodynamic jet, and demonstrated that for typical stellar and jet properties, the jets head propagates at a velocity that is at most mildly relativistic. Therefore, in this case the solution to Eq. (3) is in the transition region between the relativistic and the non-relativistic limits, and can be approximated following Bromberg et al. (2015). In order to obtain a useful analytic solution, they approximated the exact integration (shown by the dashed blue line in Fig. 1) by:

$$
t_{\mathrm{b}, \mathrm{hyd}} \simeq 6.5 R_{*, 4 R_{\odot}}\left[\left(\frac{L_{\mathrm{j}}}{L_{\mathrm{rel}}}\right)^{-2 / 3}+\left(\frac{L_{\mathrm{j}}}{L_{\mathrm{rel}}}\right)^{-2 / 5}\right]^{1 / 2} \mathrm{~s},
$$

where $L_{\mathrm{rel}}$ is the transition luminosity between a non-relativistic and a relativistic case:

$L_{\mathrm{rel}} \simeq 1.6 \times 10^{49} R_{*, 4 R_{\odot}}^{-1} M_{*, 15 M_{\odot}} \theta_{0.84}^{4}\left(\frac{3-\xi}{0.5}\right)^{7 / 5}\left(\frac{5-\xi}{2.5}\right)^{4 / 5}\left(\frac{7-\xi}{4.5}\right)^{15 / 2} \mathrm{erg} \mathrm{s}^{-1}$. 


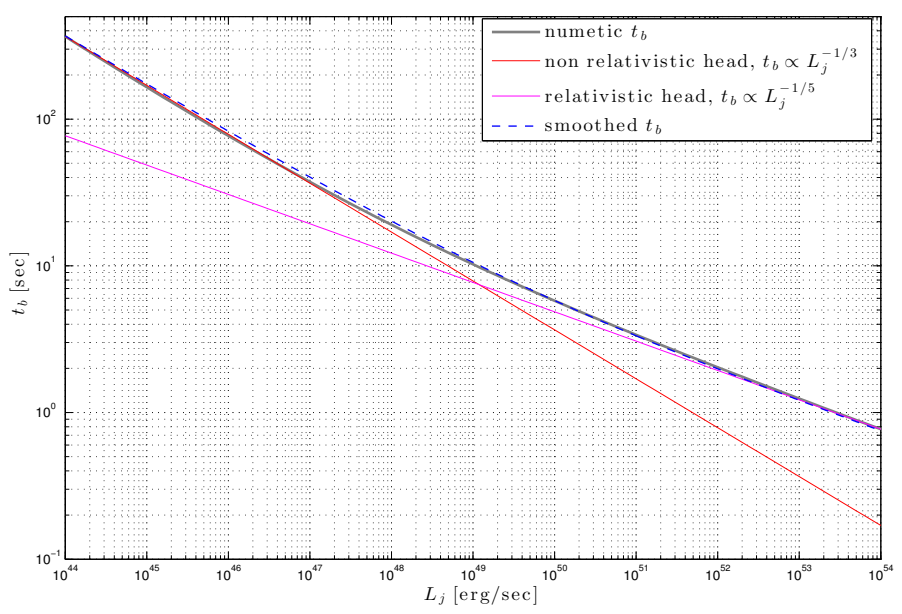

Fig. 1 The breakout time, $t_{\mathrm{b}}$, as a function of $L_{\mathrm{j}}$ calculated for a jet with an opening angle $\theta_{j}=7^{\circ}$, and a star with a mass $M_{*}=15 M_{\odot}$, radius $R_{*}=4 R_{\odot}$ and a power-law density profile $\rho \propto r^{-2.5}$. The gray solid curve tracks the exact integration of Eq. 3, the red and magenta lines show the analytic approximation for the non relativistic and the relativistic cases respectively. The dashed blue line follows the smoothed analytic solution for $t_{\mathrm{b}}$ from Eq. 7). (This figure is taken from Bromberg et al. 2015).

As canonical parameters we have used here a stellar mass of $M_{*}=15 M_{\odot}$, a stellar radius $R_{*}=4 R_{\odot}$ and we assume a power-law density profile: $\rho_{*} \propto r^{-\xi}$ with $\xi=$ 2.5. Hereafter we measure masses and radii in units of solar mass and solar radius respectively and use the subscript ' $*$ ' to denote properties of the progenitor star. For all other quantities we use the dimensionless form $A_{x} \equiv A / 10^{x}$ measured in c.g.s units. For a typical collapsar (one sided) jet luminosity of $L_{j} \sim 2 \times 10^{49} \mathrm{erg} \mathrm{s}^{-1}$, and injection angle of $\theta_{j}=7^{\circ}$ the corresponding breakout time is $t_{\mathrm{b}}\left(L_{\mathrm{rel}}\right) \simeq 9 \mathrm{~s}$.

In a Poynting flux dominated jet the situation is different. The cocoon's pressure is typically strong enough to collimate the jet before it looses causal contact with the axis. In this case the poloidal magnetic field is comparable to the toroidal field in the comoving frame of the flow, and shocks are inhibited. This leads to a smooth transition of the jet material from a free expansion state, close to the engine, to a collimated state. The jet material remains in a strong causal contact also above the collimation point. Therefore as it approaches the head, it does not shock. Moreover, it can be shown (Bromberg et al. 2014) that under these conditions the jet's proper velocity $u_{j}$ is approximately equal to the ratio of its cylindrical radius $r_{\mathrm{j}}$ and the light cylinder radius $r_{\mathrm{L}}: u_{j} \simeq r_{\mathrm{j}} / r_{\mathrm{L}}$, and the same also holds at the jet's head, $u_{\mathrm{h}} \simeq$ $r_{\mathrm{h}} / r_{\mathrm{L}}$. Therefore, the jet material gradually decelerates and becomes narrower as it approaches the head until at the head its velocity matches that of the shocked stellar material just behind the front tip of the bow shock. This deceleration and narrowing of the jet towards its head is assisted by the fact that the cocoon's pressure becomes larger closer to the head, as the bow shock is stronger there. This results in a jet head that is much narrower than in the hydrodynamic case and therefore leeds to a much 
faster propagation speed, where the head's proper speed $u_{\mathrm{h}}$ is given by (Bromberg et al. 2014):

$$
u_{\mathrm{h}} \sim \frac{r_{\mathrm{h}}}{r_{\mathrm{L}}} \sim \begin{cases}a^{1 / 5} & \left(u_{\mathrm{h}} \ll 1\right), \\ a^{1 / 6} & \left(u_{\mathrm{h}} \gg 1\right),\end{cases}
$$

where the dimensionless quantity

$$
a \equiv \frac{L_{\mathrm{j}}}{\pi \rho_{a} c^{3} r_{\mathrm{L}}^{2}}=\frac{p_{\mathrm{L}}}{\rho_{a} c^{2}} \approx 1.2 \frac{L_{50}}{\rho_{4} r_{\mathrm{L} 7}^{2}},
$$

is the ratio of the jet's magnetic pressure at the light cylinder and the ambient medium's rest mass energy density near the head 4

Therefore, a Poynting flux dominated jet becomes relativistic at a radius $R_{\text {rel }}$ deep inside the star, even with a modest power (Bromberg et al. 2014):

$$
\frac{R_{\mathrm{rel}}}{R_{*}} \simeq 1.4 \times 10^{-2}\left[L_{49.3}^{-1} M_{*, 15 M_{\odot}} R_{*, 4 R_{\odot}}^{-3} r_{L, 7}^{2}\left(\frac{3-\xi}{0.5}\right)\right]^{1 / \xi} .
$$

This implies that here only the relativistic asymptotic solution $\left(u_{\mathrm{h}} \approx a^{1 / 6}\right)$ is relevant. The corresponding breakout time is (Bromberg et al. 2015):

$$
t_{\mathrm{b}, \operatorname{mag}} \simeq 0.8 L_{49.3}^{-1 / 3} M_{*, 15 M_{\odot}}^{1 / 3} r_{L, 7}^{2 / 3}\left(\frac{0.5}{3-\xi}\right)^{2 / 3} \mathrm{~s} .
$$

This time is much shorter than the breakout time of a hydrodynamic jet with a similar luminosity.

\subsubsection{Observational Evidence for the Jet Breakout Time}

After the jet emerges from the stellar envelope it dissipates its energy at a large distance and produces the GRB. On average, the overall behavior of the prompt emission does not vary significantly during the burst (the second half of the prompt emission is rather similar to the first one). This suggests that the prompt emission arises at a more or less constant radius and not in a propagating single shell. A single shell would have expanded by a factor of $\sim 10-100$ during the duration of a burst and it is unlikely to maintain constant conditions as it emits the prompt $\gamma$-ray emission over such a wide range of radii. This implies, in turn, that the GRB activity follows the central engine's activity (Sari and Piran 1997), and that the GRB lasts as long as the central engine is active. Therefore, within the Collapsar model, the observed GRB duration (usually denoted by $T_{90}$, which measures the time over which the central $90 \%$ of the prompt photon counts are detected) is the difference between the engine operation time, $t_{e}$, and the breakout time, $t_{\mathrm{b}}$, namely $T_{90}=t_{e}-t_{\mathrm{b}}$ (not accounting for the cosmological time dilation here).

The breakout time essentially serves as a threshold time: a regular GRB is formed only if $t_{e}>t_{\mathrm{b}}$. Bromberg et al. (2012) have shown that in such a case one would

\footnotetext{
4 This analysis does not account for 3D effects that can slow down the head's propagation speed (Bromberg \& Tchekhovskoy 2015, in prep).
} 
expect a plateau in the duration distribution of GRBs, $d N_{\mathrm{GRB}} / d T_{90}$, at durations that are shorter than $t_{\mathrm{b}}$. The logic behind this is as follow. At the time when the jet's head breaches the edge of the star, it is already disconnected from the engine and cannot transmit information backward to the engine. In other words, the engine cannot "tell" when the jet breaks out of the star and we do not expect that $t_{e}$ and $t_{\mathrm{b}}$ will be strongly related to each other. In fact, for a given $t_{\mathrm{b}}$ we expect to have a distribution of engine activity times, where some are shorter $\left(t_{e}<t_{\mathrm{b}}\right)$ and some are longer $\left(t_{e}>t_{\mathrm{b}}\right)$ than $t_{\mathrm{b}}$. In this case the probability of observing a GRB with duration $T_{90}$ is equal to the probability that the engine will work for a time $t_{e}=T_{90}+t_{\mathrm{b}}: P_{\mathrm{GRB}}\left(T_{90}\right) \equiv P_{e}\left(t_{e}=\right.$ $\left.T_{90}+t_{\mathrm{b}}\right)$. This probability has a simple description in two limits:

$$
P_{\mathrm{GRB}}\left(T_{90}\right) \approx\left\{\begin{array}{cc}
P_{e}\left(t_{\mathrm{b}}\right) & \left(T_{90} \ll t_{\mathrm{b}}\right), \\
P_{e}\left(T_{90}\right) & \left(T_{90} \gg t_{\mathrm{b}}\right) .
\end{array}\right.
$$

Now, if there is a dominant population of GRBs with a typical $t_{\mathrm{b}}$, then at short durations $P_{\mathrm{GRB}}\left(T_{90}\right) \rightarrow P_{e}\left(t_{\mathrm{b}}\right)=$ const, we expect to get a plateau at durations $T_{90} \ll t_{\mathrm{b}}$.

Figure 2 depicts the duration distribution, $d N_{\mathrm{GRB}} / d T_{90}$, of BATSE 5 (2100 GRBs), Fermi-GBM ${ }^{6}$ (1310 GRBs) and Swif $7^{7}$ (800 GRBs). To fit a plateau in each data set Bromberg et al. (2015) looked for the maximal number of bins that are consistent with a plateau at a confidence level $\leq 95 \%(2 \sigma)^{8}$. The extent of the best fitted plateaus is $5-25 \mathrm{~s}$ in the BATSE data $\left(7.19 / 4 \chi^{2} /\right.$ d.o.f $), 2.5-17 \mathrm{~s}$ in the Fermi-GBM data $(10 / 5$ $\chi^{2} /$ d.o.f), and 1-20 s in the Swift data (15.85/9 $\chi^{2} /$ d.o.f). Bromberg et al. (2015) accounted for three free parameters in the fit: the height of the plateau and the two opposite ends of the plateau line. The differences between the maximal durations of the plateaus can be mostly attributed to the different sensitivity and triggering algorithms of the different detectors.

At short durations, the plateau is concealed by the increasing number of nonCollapsar ("short") GRBs having a typical duration of less than a few seconds (Bromberg et al. 2013). As non-Collapsars have on-average harder spectra than Collapsars (e.g. Kouveliotou et al. 1993), the relative number of non-Collapsars can be reduced by choosing a hardness threshold (for each sample) and selecting only the events that are softer than this threshold. This should lead to a less prominent "bump" at short duration. If the plateau is indeed an intrinsic property of the (softer) Collapsars duration distribution, it should extend to shorter durations in a softer subsample. To examine this effect they selected in each sample all the events that are softer than the median hardness of long GRBs $\left(T_{90}>20 \mathrm{~s}\right)$ in the sample (see Bromberg et al. 2013 for further details). Figure 3 shows the duration distribution of the soft GRB subsamples. The plateaus indeed extend to much shorter durations than in the complete samples, supporting our hypothesis. The extent of the best fitted plateaus is $0.4-25 \mathrm{~s}$

\footnotetext{
5 http://gammaray.msfc.nasa.gov/batse/grb/catalog/current/ from April 21, 1991 until August 17, 2000.

6 http://heasarc.gsfc.nasa.gov/W3Browse/fermi/fermigbrst.html, from July 17, 2008 until February 14, 2014.

7 http://swift.gsfc.nasa.gov/archive/grb_table/, from December 17, 2004 until February 14, 2014.

8 The confidence level is defined here as $\int_{0}^{\chi^{2}} P(x, v) d x$, where $P\left(\chi^{2}, v\right)$ is the probability density function of $\chi^{2}$ with $v$ degrees of freedom (Press et al. 1992),
} 


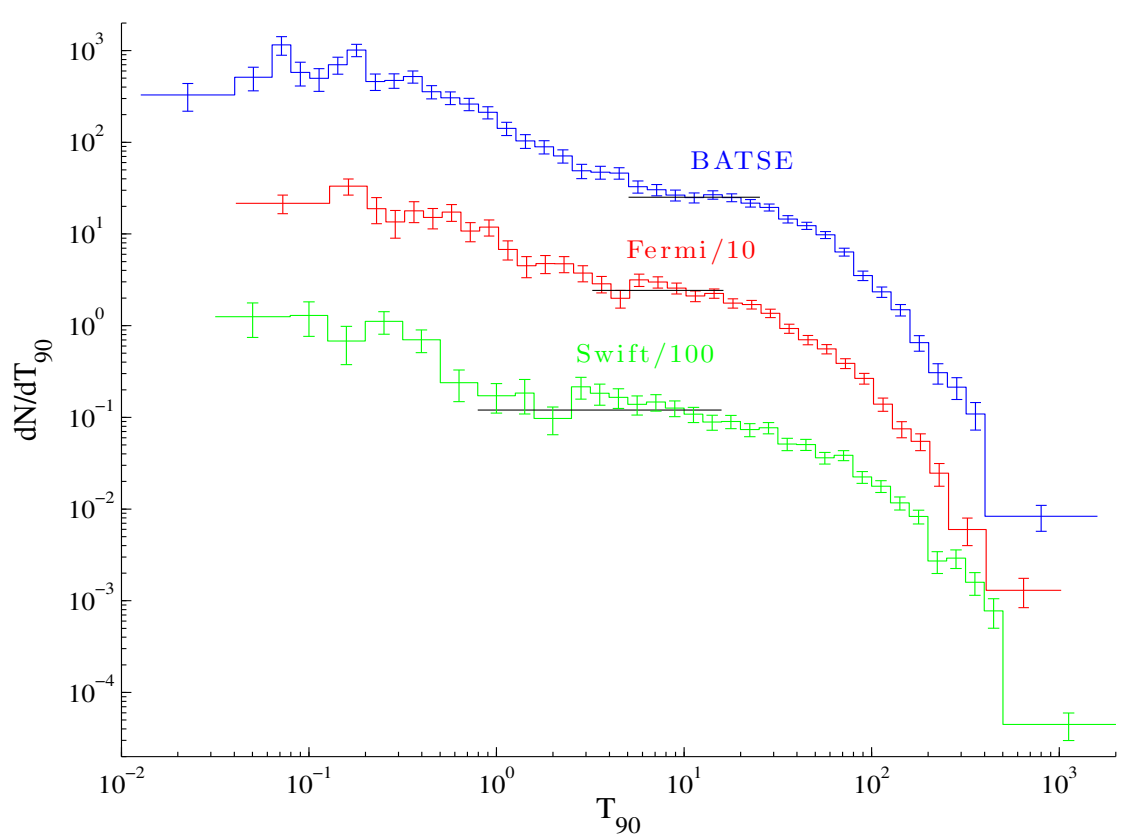

Fig. 2 The duration distribution, $d N_{\mathrm{GRB}} / d T_{90}$ of BATSE (blue), Fermi (red) and Swift (Green) GRBs. The different curves are shifted in order to avoid overlap. The data bins are evenly spaced in logarithmic scale with $\Delta \log \left(T_{90}\right)=0.1$. Bins with less than 5 events are combined with their neighbors in order to achieve statistical significance. The black horizontal lines mark the bins that fit a plateau at a confidence interval up to $2 \sigma$. (This figure is taken from Bromberg et al. 2015,

in the BATSE data $\left(20.75 / 12 \chi^{2} /\right.$ d.o.f $), 0.4-17 \mathrm{~s}$ in the Fermi-GBM data $(8.7 / 10$ $\chi^{2} /$ d.o.f) and $0.2-20 \mathrm{~s}$ in the Swift data $\left(9.04 / 8 \chi^{2} /\right.$ d.o.f).

Taking a median redshift of $z \simeq 2$ for Swift GRBs and $z \simeq 1$ for Fermi-GBM and BATSE bursts, Bromberg et al. (2015) find that in the GRBs' rest frame, these plateaus extend up to intrinsic durations of 7-12 s, consistent with the results obtained by Bromberg et al. (2012). Note that the actual $t_{\mathrm{b}}$ may be somewhat longer than the duration that marks the end of the plateau, but it cannot be shorter. We use the duration interval of 7-12 s as our best estimate for the typical $t_{\mathrm{b}}$.

\subsubsection{Implications: the Jet Composition at Early Times}

Eqs. (77), (8) and (12) use parameter values inferred from typical GRB jets, after accounting for the jet opening angle (e.g., Bloom et al.|2003). From these equations it can be seen that a breakout time of 7-12 s is consistent with that expected for a hydrodynamic jet from a WR star with a radius of $\sim(3-6) R_{\odot}$. On the other hand, these breakout times are too long for typical parameters expected for a Poynting dominated jet. To account for these breakout times, the light cylinder of the compact object at the base of the jet should be of the order of $r_{\mathrm{L}} \simeq(2.5-5) \times 10^{8} \mathrm{~cm}$, corresponding to an angular frequency of $\Omega_{m} \simeq 60-120 \mathrm{rad} / \mathrm{s}$ at the base of the jet. Such a frequency is too low to allow the engine to power a typical GRB jet (Bromberg et al. 2015). 


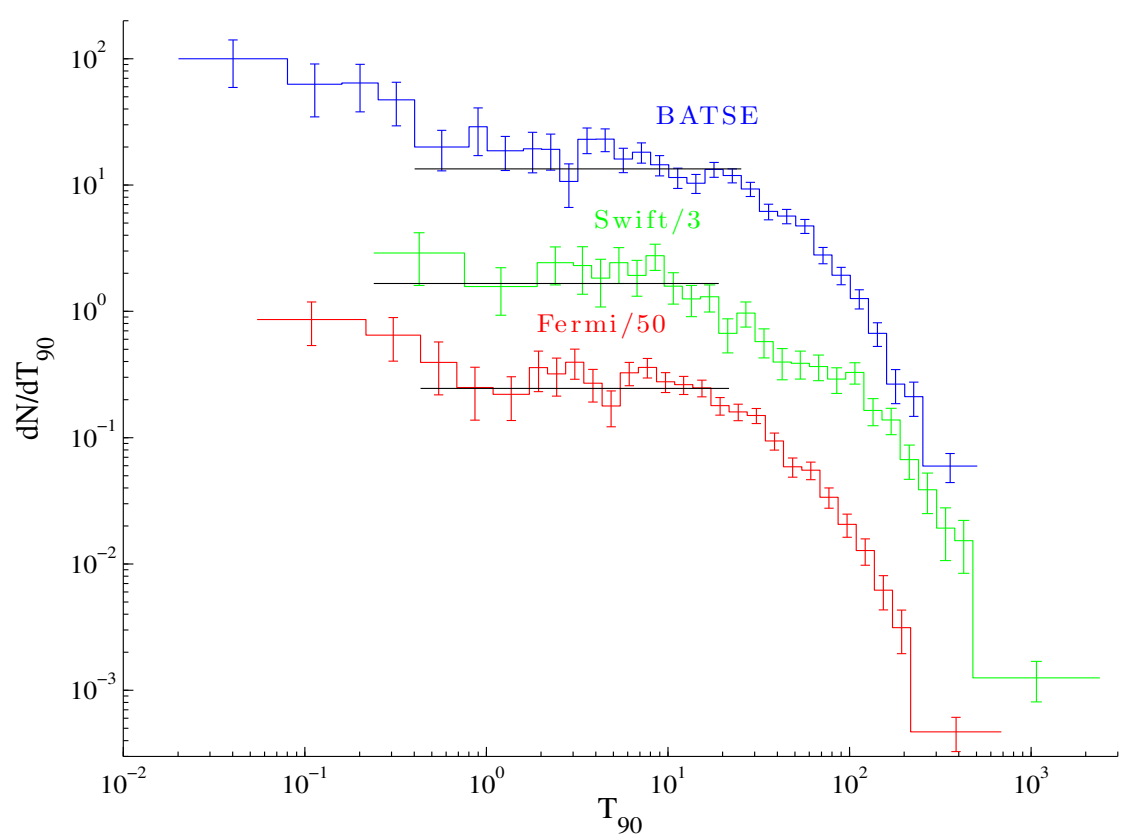

Fig. 3 The duration distribution, $d N_{\mathrm{GRB}} / d T_{90}$ of the soft GRBs. The analysis is the same as in Figure 2 only the data from each satellite contains only events that are softer than the median hardness of the long GRBs with durations $T_{90}>20 \mathrm{~s}$. For BATSE, this corresponds to GRBs having a hardness ratio $H R_{32}<2.6$, for Fermi the GRBs have a power law spectral index $<-1.5$, and for Swift the GRBs have a power law spectral index $<-1.7$. The analysis here updates the analysis in Bromberg et al. (2013) using a more complete recent sample. (This figure is taken from Bromberg et al. 2015)

The conclusion arising from this analysis is that during most of its propagation within the star the jet has a low magnetization and it propagates as a hydrodynamic jet (unless 3D effects significantly increase $t_{\mathrm{b}, \mathrm{mag}}$ ). This result leads to some interesting implications for the properties of long GRB central engines and the conditions at the base of the jets. One possibility is that the jet is launched hydrodynamically at the source. The most probable process for that is neutrino-anti-neutrino annihilation above the rotational axis of the central engine (e.g. Eichler et al. 1989; Levinson and Eichler 1993). This scenario can work only if the accretion rate is $\gtrsim 0.1 M_{\odot} \mathrm{s}^{-1}$, so that neutrino emission is large enough to power the observed jets (Kawanaka et al. 2013; Levinson and Globus 2013). The high accretion rate must be sustained throughout the entire duration of the GRB, which can last from tens to hundreds of seconds. Though a duration of $\lesssim 30$ s seems to be consistent with such a model (e.g. Lindner et al. 2010), its seems unlikely to be capable of powering longer duration GRBs.

A second possibility is that the jet is launched Poynting flux dominated but it dissipates most of its magnetic energy close to the source, and it then propagates as a hydrodynamic jet. An appealing process for such efficient dissipation is the kink instability (Lyubarskij 1992; Eichler 1993, Spruit et al. 1997, Begelman|1998, Lyubarskii 1999: Giannios and Spruit 2006). Bromberg et al. (2014), however, have shown via analytic considerations, that collapsar jets are less likely to be disrupted by the kink 
instability. Thus a different process, possibly internal to the jet, may be needed to dissipate the jet energy. A definite answer will be obtained only via 3D numerical simulations, which are underway. In one such work, Bromberg \& Tchekhovskoy (2015, in prep.) show that indeed kink instability is unlikely to disrupt a typical collapsar jet. Nevertheless, kink modes can grow internally in the jet and lead to efficient dissipation of the magnetic energy via reconnection of the magnetic field lines without compromising the jets's integrity. The outcome of such dissipation is a jet with an equipartition between thermal and magnetic energy, which propagates more or less like a hydrodynamic jet.

A third possibility is that the jet changes its character with time. Our conclusion concerning the jet composition applies only to the initial phase, while its head is still within the stellar envelope. This phase, which lasts $\sim 10 \mathrm{~s}$, must be predominantly hydrodynamic. Once the jet has breached the star it can be Poynting flux dominated. This would require a more complicated central engine that switches from one mode to another. While this seems contrived, remarkably, some magnetar models suggest such a possibility (Metzger et al. 2011). One can also imagine accretion disk models that initially cool via neutrinos and later on as the accretion rate decreases, become Poynting flux dominated (Kawanaka et al. 2013). However, all such models require some degree of coincidence as the central engine does not receive any feedback from the propagating jet and there is no a priori reason that the transition from one composition to the other would take place just at the right stage.

\subsection{Jet steady State Acceleration}

Magnetic acceleration and thermal acceleration are the two main competing mechanisms for the acceleration of GRB jets or outflows. Thus, the acceleration mechanism is tightly related to the outflow composition and in particular its degree of magnetization, which is both highly uncertain and of great interest. In other sources of relativistic jets or outflows, there are currently better constraints on the composition. Pulsar winds are almost certainly Poynting flux dominated near the central source. This most likely also holds for active galactic nuclei (AGN) and tidal disruption events, as in these sources the central accreting black hole is supermassive, and therefore even close to it the Thompson optical depth, $\tau_{T}$, may not be high enough for thermal acceleration by radiation pressure (the main competition to magnetic acceleration) to work efficiently (e.g., Ghisellini 2012). In GRBs or micro-quasars, however, thermal acceleration could also work (since $\tau_{T} \gg 1$ is possible, or even likely close enough to the source), and the dominant acceleration mechanism is less clear.

First, let us consider the thermal acceleration of a steady, axisymmetric, and unmagnetized flow that is initially relativistically hot with $p \gg \rho c^{2}$. Let the jet cross section be $\Sigma \propto r^{2}$ where $r$ is its cylindrical radius. The relativistic equation of state implies $p \propto \rho^{4 / 3}$, while mass and energy conservation $\operatorname{read} \Gamma \rho c \Sigma=$ const and $\Gamma^{2}\left(\rho c^{2}+\right.$ $4 p) c \Sigma=$ const, respectively (where we have assumed a relativistic velocity, $\beta=$ $v / c \approx 1$ ). The ratio of the two last expressions gives the Bernoulli equation - the total energy per unit rest energy (which is conserved without any significant energy losses from the system), $\left(1+4 p / \rho c^{2}\right) \Gamma=$ const. As long as the flow is relativisti- 
cally hot $\left(p \gg \rho c^{2}\right)$ it accelerates as $\Gamma \propto \rho / p \propto \rho^{-1 / 3} \propto \Sigma^{1 / 2} \propto r$. This reproduces the familiar result for a spherical or conical flow for which $\Gamma \propto r \propto z$, i.e. the Lorentz factor grows linearly with the distance $z$ from the central source. Therefore, thermal acceleration is relatively fast, efficient and robust.

Let us now do a similar simple analysis for a cold and initially highly-magnetized flow, with $\sigma_{0}=B_{0}^{2} / 4 \pi \rho_{0} c^{2} \gg 1$ (e.g., Komissarov 2011). Let the flow be steady, axisymmetric, and ideal MHD (i.e. without magnetic dissipation). Let us consider the flow between two magnetic flux surfaces defined by $r$ and $r+\delta r$ (which are both functions of $z$ ). Flux freezing (ideal MHD) implies that the poloidal and tangential magnetic field components scale as $B_{p} \propto 1 / r \delta r$ and $B_{\phi} \propto 1 / \delta r$, respectively, in the lab frame. Therefore, the tangential field component rapidly dominates far from the source, so that $B \approx B_{\phi} \approx \Gamma B^{\prime}$ where $B^{\prime}$ in the magnetic field in the comoving frame of the outflowing plasma (in which the electric field vanishes). Altogether this gives $B=\Gamma B^{\prime} \propto 1 / \delta r$. Mass and energy conservation read $\Gamma \rho c \Sigma=$ const and $\Gamma^{2}\left(\rho c^{2}+\right.$ $\left.B^{\prime 2} / 4 \pi\right) c \Sigma=$ const, respectively, where $\Sigma \propto r \delta r$. Their ratio implies a total energy per unit rest energy of $(1+\sigma) \Gamma=\left(1+\sigma_{0}\right) \Gamma_{0}=\Gamma_{\max }$ where $\sigma=B^{\prime 2} / 4 \pi \rho c^{2} \propto r / \Gamma \delta r$ is the magnetization parameter. Therefore, this results in the following Lorentz factor evolution:

$$
\frac{\Gamma}{\Gamma_{0}}=1+\sigma_{0}\left(1-\frac{\delta r_{0}}{r_{0}} \frac{r}{\delta r}\right), \quad \frac{\Gamma}{\Gamma_{\max }}=1-\left(1-\frac{\Gamma_{0}}{\Gamma_{\max }}\right) \frac{\delta r_{0}}{r_{0}} \frac{r}{\delta r} .
$$

This immediately implies that for a conical (or spherical) flow, in which $\delta r \propto r$ and $\delta r / r=\delta r_{0} / r_{0}$, the Lorentz factor essentially remains constant, $\Gamma \approx$ const, and the flow hardly accelerates. This result can be understood by simple energy considerations. As long as there is no expansion along the direction of motion, the volume of a fluid element scales as $\propto r^{2}$ while its magnetic energy density scales as $\propto B^{2} \propto r^{-2}$, implying a constant magnetic energy and no conversion into kinetic energy.

More generally, Eq. 14 implies that in order for the flow to accelerate, $r / \delta r$ must decrease, i.e. streamlines must diverge faster than conical. For power-law streamlines, $z=z_{0}\left(r / r_{0}\right)^{\alpha}=z_{0}\left[(r+\delta r) /\left(r_{0}+\delta r_{0}\right)\right]^{\alpha}$, one has $r / \delta r=r_{0} / \delta r_{0}$ so there is still no acceleration. If one allows the power law index to vary with $r_{0}=r\left(z_{0}\right)$, i.e. $\alpha=\alpha\left(r_{0}\right)$, then one finds $\delta r / r=\left(\delta r_{0} / r_{0}\right)\left[1-r_{0} \alpha^{\prime}\left(r_{0}\right) \alpha^{-2}\left(r_{0}\right) \ln \left(z / z_{0}\right)\right]$, and the condition for acceleration becomes $\alpha^{\prime}=d \alpha / d r_{0}<0$. Altogether one can see that such steady-state, axisymmetric ideal MHD acceleration is quite delicate and requires a very particular configuration of the magnetic field lines. Satisfying this requirement is not trivial, and in particular it requires lateral causal contact across the jet.

A key open question regarding outflows that start out highly magnetized near the central source is how they convert most of their initial electromagnetic energy to other forms, namely bulk kinetic energy or the energy in the random motions of the particles that also produce the radiation we observe from these sources. It is suggested by observations of relevant sources, such as AGN, GRBs or pulsar wind nebulae that the outflow magnetization is rather low at large distances from the source. This is the essence of the well-known $\sigma$ problem - how to transform from $\sigma \gg 1$ near the source to $\sigma \ll 1$ very far from the source.

It has been shown early on that a highly magnetized steady spherical flow accelerates only up to an asymptotic Lorentz factor $\Gamma_{\infty} \sim \sigma_{0}^{1 / 3}$, and magnetization $\sigma_{\infty} \sim \sigma_{0}^{2 / 3}$ 
(Goldreich and Julian 1970) where $\sigma_{0} \gg 1$ is the initial value of the magnetization parameter $\sigma$, implying that most of the energy remains in electromagnetic form (a Poynting flux dominated flow). This is valid for any such unconfined flow, i.e. where the external pressure is effectively negligible. A sufficiently large external pressure can help collimate and accelerate the flow. It has been found (Lyubarsky 2009. 2010b Komissarov et al. 2009) that for a power law external pressure profile, $p_{\text {ext }} \propto z^{-\kappa}$, the collimation and acceleration can proceed in two distinct regimes.

For $\kappa>2$, the weak confinement regime, the external pressure drops fast enough such that the flow loses lateral causal contact while it is still highly magnetized, and from that point on it becomes conical and essentially stops accelerating. This collimation-induced acceleration increases $\Gamma_{\infty}$ and decrease $\sigma_{\infty}$ by up to a factor of $\sim \theta_{\mathrm{j}}^{-2 / 3}$ compared to the unconfined (quasi-spherical) case, where $\theta_{\mathrm{j}}$ is the asymptotic jet half-opening angle. This arises because lateral causal contact in the jet is maintained as long as $\theta_{\mathrm{j}}$ does not exceed the Mach angle, $\theta_{\mathrm{j}} \lesssim \theta_{\mathrm{M}} \sim \sigma^{1 / 2} / \Gamma$, where energy conservation implies $\sigma \Gamma \sim \sigma_{0}$ (for $\sigma_{0} \gg 1$ and $\Gamma_{0} \sim 1$ ) as long as the flow remains highly magnetized $(\sigma \gg 1)$.

For $\kappa \leq 2$, the strong confinement regime, the external pressure drops slowly enough that the jet maintains lateral causal contact throughout its collimation-induced acceleration process. In this case about half of the initial magnetic energy is converted into kinetic energy and the flow bacomes only mildly magnetized, $\sigma_{\infty} \sim 1$, while the Lorentz factor approaches its maximal possible value, $\Gamma_{\infty} \sim \sigma_{0}$. In this regime the collimation and acceleration proceed as $\Gamma \propto r \propto z^{\kappa / 4}$ and the jet remains narrow, $\Gamma_{\infty} \theta_{\mathrm{j}} \sim 1$.

The main problem with this picture, however, is that even under the most favorable conditions the asymptotic magnetization is $\sigma_{\infty} \geq 1$, which does not allow efficient energy dissipation in internal shocks within the outflow (Lyubarsky 2009 2010b, Komissarov et al. 2009). It has been found (Tchekhovskoy et al. 2010; Komissarov et al. 2010) that a sudden drop in the external pressure, as may occur when a GRB jet exits its progenitor star, can result in a sudden additional acceleration that can lead to $\Gamma_{\infty} \theta_{\mathrm{j}} \gg 1$ as inferred in GRBs, but still with $\sigma_{\infty} \geq 1$.

These important limitations of the "standard" steady, axisymmetric and nondissipative (or ideal MHD) magnetic acceleration have, on the one hand, led to the suggestion that the jets might remain Poynting flux dominated at large distances from the source and the observed emission is the result of magnetic reconnection events rather than internal shocks (Blandford 2002, Lyutikov and Blandford 2003, Lyutikov 2006). On the other hand, other models suggested increasing the acceleration efficiency by relaxing one of the standard assumptions, such as axi-symmetry - leading to non-axisymmetric instabilities that randomize the magnetic field orientation (Heinz and Begelman 2000). Since a highly tangled magnetic field effectively behaves like a relativistic fluid (with an adiabatic index of 4/3) this leads to efficient acceleration, similar to thermal acceleration of relativistic outflows. What is more, both the kink instability mentioned above (Drenkhahn and Spruit 2002), as well as other instabilities (such as the Kruskal-Schwarzschild instability in a striped wind; Lyubarsky 2010a) can lead to magnetic reconnection, i.e. gradual magnetic dissipation, which in turn enhances the acceleration due to the conversion of magnetic to thermal energy, where the thermal pressure efficiently accelerates the outflow. 

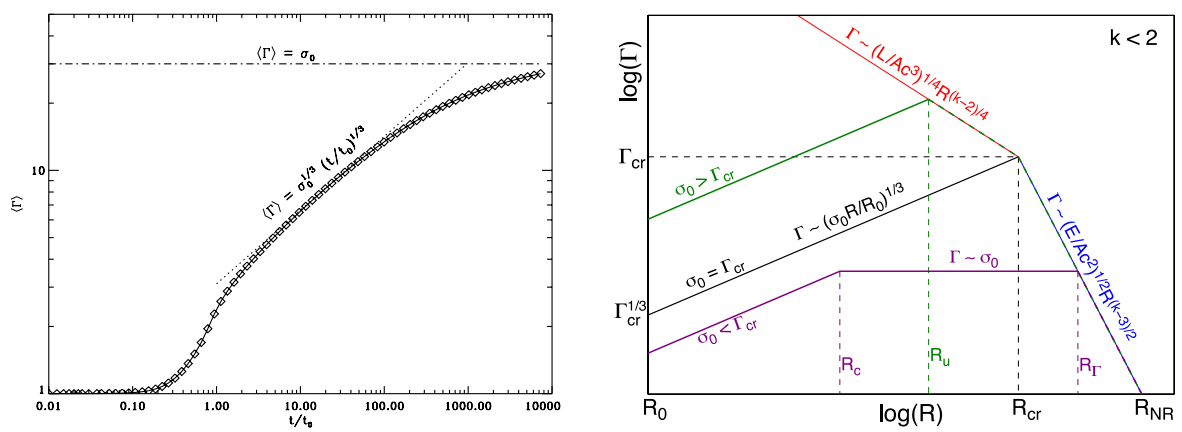

Fig. 4 Left: Test case for impulsive magnetic acceleration: the energy-weighted mean Lorentz factor $\langle\Gamma\rangle$ of a finite cold shell of plasma initially uniform (with width $l_{0}$, rest mass density $\rho_{0}$ and magnetic field $\left.B_{0}\right)$, highly magnetized $\left(\sigma_{0}=B_{0}^{2} / 4 \pi \rho_{0} c^{2} \gg 1 ; \sigma_{0}=30\right.$ was used here) and at rest, whose back leans against a conducting "wall" while its front faces vacuum, versus the time $t$ in units of the shell's initial fast magnetosonic crossing time $t_{0} \approx l_{0} / c$. The analytic expectations (dotted and dashed-dotted lines) and the results of numerical simulations (diamond symbols joined by a solid line) are in very good agreement. (This Figure is taken from Granot et al.2011). Right: Evolution of the typical (or energy-weighted average) Lorentz factor $\Gamma$ with the distance $R \approx c t$ from the central source, for a finite shell similar to that described in the left panel, but for a spherical shell propagating into an external medium with a power-law density profile, $\rho_{\mathrm{ext}}=A R^{-k}$. (This figure is taken from Granot 2012a).

\subsection{Impulsive Magnetic Acceleration}

Replacing the usual steady-state assumption by strong time-dependence is a natural alternative. This impulsive regime was sparsely studied, and mainly in the nonrelativistic case (Contopoulos 1995). Recently, a new impulsive magnetic acceleration mechanism was found that operates in the relativistic case (Granot et al. 2011), which can be much more efficient than magnetic acceleration in steady flows, and can lead to low magnetizations, $\sigma \ll 1$, thus enabling efficient dissipation in internal shocks. This qualitatively different behavior of impulsive outflows can be very relevant for GRBs, as well as for other relativistic jet sources such as tidal disruptions or flares in AGN or micro-quasars, or even giant flares in soft gamma repeaters (SGRs, thought to be magnetars), which also triggered renewed interest in this topic (e.g., Levinson 2010, Lyutikov 2011, Granot 2012a|b, Komissarov|2012).

Figure 4 (left panel) shows results for an impulsive magnetic acceleration test case: a cold, initially uniform plasma shell (of with width $l_{0}$, rest mass density $\rho_{0}$ and magnetic field $\left.B_{0}\right)$, highly magnetized $\left(\sigma_{0}=B_{0}^{2} / 4 \pi \rho_{0} c^{2} \gg 1\right)$ and at rest, with a conducting "wall" at its back and vacuum in front of it. A strong, self-similar rarefaction wave forms at its front (vacuum interface) and propagates towards its back, reaching the wall at $t=t_{0} \approx l_{0} / c$. By this time the shell's energy-weighted mean Lorentz factor and magnetization are $\langle\Gamma\rangle \sim \sigma_{0}^{1 / 3}$ and $\langle\sigma\rangle \sim \sigma_{0}^{2 / 3}$. At $t>t_{0}$ the shell detaches from the wall, keeps an almost constant width $\left(l \approx 2 l_{0}\right)$ and accelerates as $\langle\Gamma\rangle \sim \sigma_{0} /\langle\sigma\rangle \sim\left(\sigma_{0} t / t_{0}\right)^{1 / 3}$ up to the coasting time $t_{c}=\sigma_{0}^{2} t_{0}$. At $t>t_{c}$ the shell coasts at $\langle\Gamma\rangle \sim \sigma_{0}$, its width grows $\left(l / 2 l_{0} \sim t / t_{c}\right)$ and its magnetization rapidly decreases $\left(\langle\sigma\rangle \sim t_{c} / t\right)$, leading to complete conversion of magnetic to kinetic energy that allows strong internal shocks to form that can lead to large radiative efficiencies. 


\subsection{Interaction with the External Medium and the Reverse Shock}

Let us now consider the evolution of a similar shell in spherical geometry that propagates into an external medium with a power-law density profile, $\rho_{\mathrm{ext}}=A R^{-k}$, following Granot (2012a). The main results are shown in the right panel of Figure 4 The initial shell magnetization $\sigma_{0}$ and density $\rho_{0} \propto 1 / \sigma_{0}$ are allowed to vary while keeping fixed the values of the initial time or length scale $\left(t_{0} \approx R_{0} / c\right.$ or $\left.R_{0}\right)$, energy $\left(E \sim L t_{0} \approx L R_{0} / c\right.$ or power $\left.L\right)$, and external density $(k<2$ in this figure, and $A$ or $\left.\rho_{\text {ext }}\left(R_{0}\right)=A R_{0}^{-k}\right)$, which imply fixed $\Gamma_{\text {cr }} \sim\left(f_{0} \sigma_{0}\right)^{1 /(8-2 k)}$ where $f_{0}=\rho_{0} / \rho_{\text {ext }}\left(R_{0}\right)$ and $R_{\mathrm{cr}} \sim R_{0} \Gamma_{\mathrm{cr}}^{2}$. Shown are the two dynamical regimes most relevant for GRBs. The purple line shows regime I ( $1<\sigma_{0}<\Gamma_{\text {cr }}$ or a sufficiently low external density) where the shell initially expands as if into vacuum (as described in the left panel) and only after becoming kinetically dominated and expanding radially is it significantly decelerated by the external medium through a strong relativistic reverse shock, which can produce a bright emission that peaks on a timescale larger than the duration of the prompt GRB emission (the familiar low- $\sigma$ "thin shell"; Sari and Piran 1995). Eventually, most of the energy is transfered to the shocked external medium and the flow approaches the Blandford and McKee (1976) self-similar solution.

In regime II $\left(1<\Gamma_{\mathrm{cr}}<\sigma_{0}<\Gamma_{\mathrm{cr}}^{3(4-k) / 2}\right)$, depicted by the green line in the right panel of Figure 4 , the shell is significantly affected by the external medium while it is still Poynting dominated (at $R>R_{u} \sim R_{0}\left(f_{0} \sigma_{0}^{-1 / 3}\right)^{3 /(10-3 k)}$ ), thus suppressing the reverse shock (which is either non-existent or very weak). The shell remains highly magnetized and gradually transfers its energy to the shocked external medium through $p d V$ work across the contact discontinuity up to $R_{\mathrm{cr}}$, after which the flow approaches the Blandford-McKee solution. In this regime no significant reverse shock emission is expected, and the onset of the afterglow (i.e. the peak of the emission from the shocked external medium) is expected to be on a timescale comparable to the prompt GRB duration (i.e. a high- $\sigma$ "thick shell").

In addition, there are other regimes not shown in this figure, but all of the regimes are mapped in the relevant parameter space in Figure 5 . In regime III $\left(1<\Gamma_{\mathrm{cr}}^{3(4-k) / 2}<\right.$ $\left.\sigma_{0}\right)$ the external density is high enough that there is no impulsive acceleration stage where $\langle\Gamma\rangle \propto R^{1 / 3}$, and instead $\langle\Gamma\rangle \sim \sigma_{0} /\langle\sigma\rangle \propto R^{(k-2) / 4}$ at $R_{0}<R<R_{\mathrm{cr}} \sim R_{\mathrm{dec}}$, and then approaches the Blandford-McKee solution (its observational signatures are expected to be similar to regime II). In regime IV $\left(\Gamma_{\mathrm{cr}}<1\right)$ the external density is so high that the flow remains Newtonian all along (as might happen while the GRB jet is propagating inside a massive star progenitor). There is also an "exotic" regime II* that exists only in a highly stratified external medium $(10 / 3<k<4)$.

Under realistic conditions, GRB variability times are in practice typically large enough that the flow should first undergo quasi-steady collimation-induced acceleration that saturates, and only later the impulsive acceleration kicks in and operates until the flow becomes kinetically dominated (see Figure 6). Moreover, one typically expects the outflow from the central source to consist of many sub-shells rather than a single continuous shell. The effects of such multiple sub-shells in the outflow can be important, and the collisions between them may provide efficient energy dissipation that can power the GRB emission (Granot 2012b, Komissarov 2012). They may 

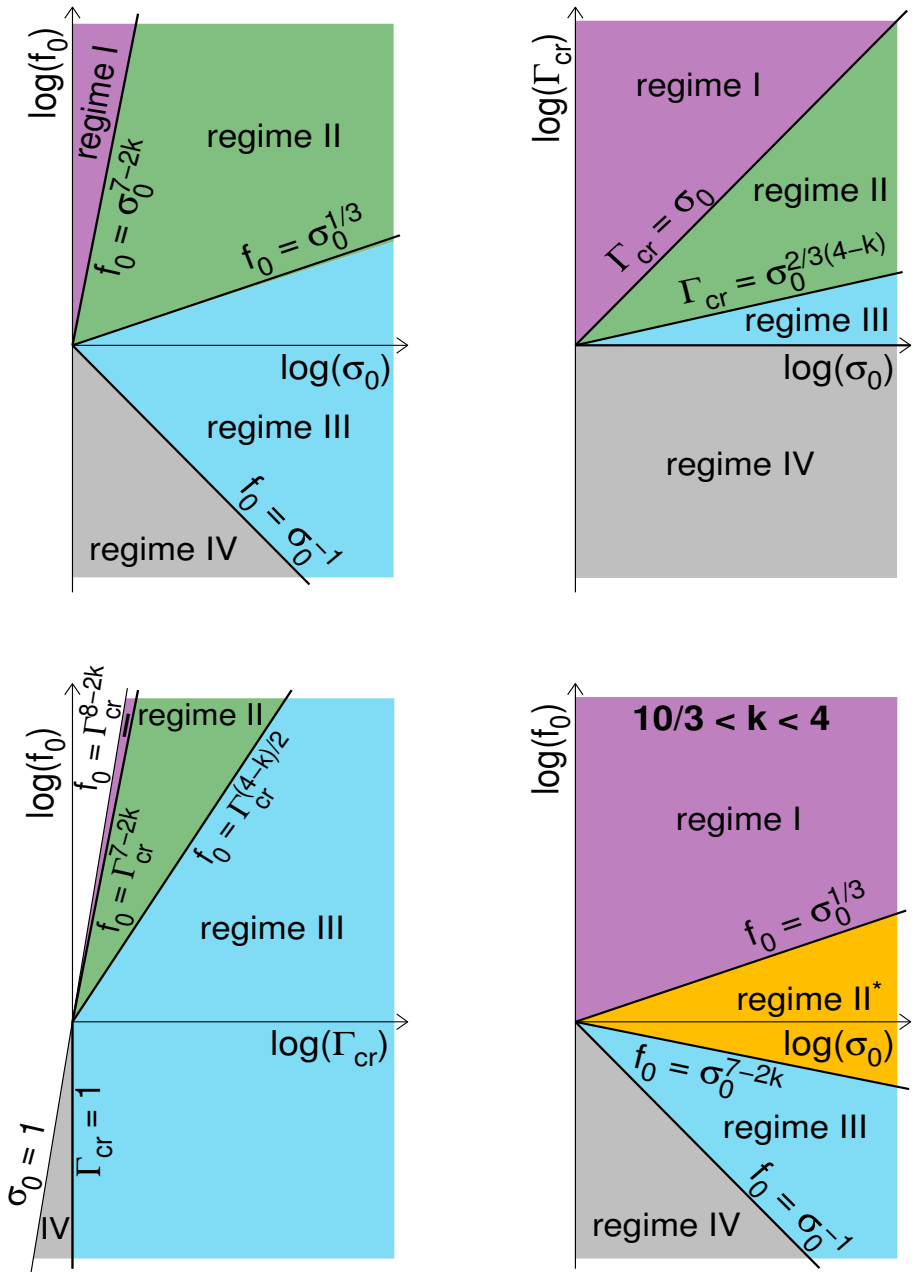

Fig. 5 Phase space diagrams of the different dynamical regimes: in the $f_{0}-\sigma_{0}$ plane for $k<10 / 3$ (top left panel), $\Gamma_{\mathrm{cr}}-\sigma_{0}$ plane for $k<10 / 3$ (top right panel), $f_{0}-\Gamma_{\mathrm{cr}}$ plane for $k<10 / 3$ (bottom left panel), and in the $f_{0}-\sigma_{0}$ plane for $10 / 3<k<4$ (bottom right panel). Each regime is labeled and denoted by a different color, and the borders between the different regimes are indicated by labeled thick black lines. (This figure is taken from Granot 2012a )

also allow a low- $\sigma$ "thick shell", i.e. a strong relativistic reverse shock peaking on a timescale comparable to the prompt GRB emission, which is not possible for a single shell. For a long-lived source (e.g. AGN) with initial sub-shell widths $l_{0}$ and separations $l_{\text {gap }}$, each sub-shell can expand by a factor of $1+l_{\text {gap }} / l_{0}$. Its magnetic energy decreases by the same factor (where $\sigma_{\infty} \sim l_{0} / l_{\text {gap }}$ ), and may be converted to kinetic or internal energy, or radiation. For a finite source activity time, the merged shell can still expand further and convert more magnetic energy into other forms (even without interaction with an external medium). Important related points that warrant further study are the transition from quasi-steady collimation-induced acceleration to 


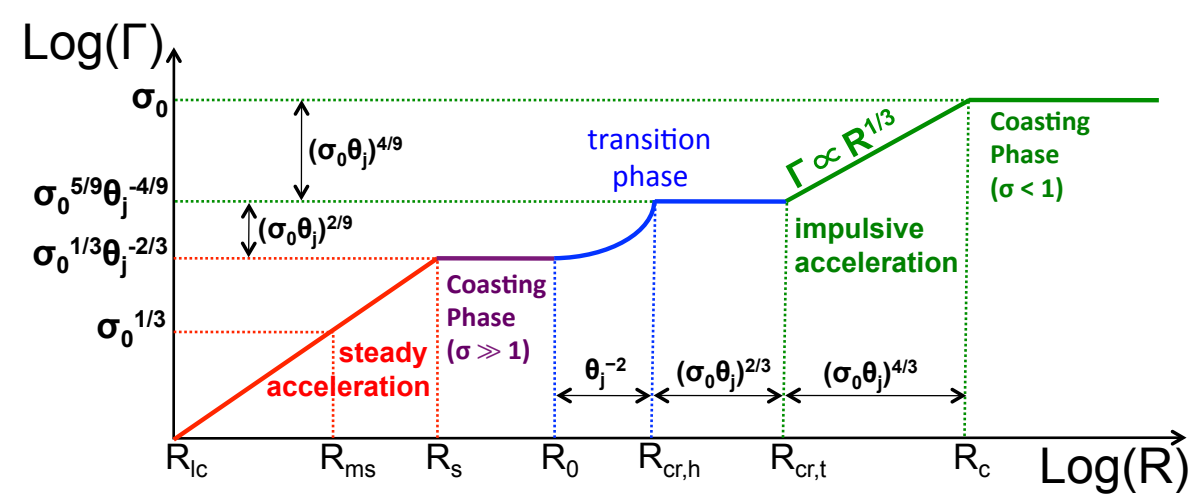

Fig. 6 An illustration of the expected transition from (quasi-) steady collimation-induced acceleration near the central source (in red) to impulsive acceleration further away from the source (in green). The energy weighted mean Lorentz factor $\Gamma$ is shown against the distance $R$ from the central source, and a few critical radii and Lorentz factors are indicated following the notations of Granot et al. (2011) and Granot (2012a).

impulsive acceleration, both in a single shell and in multiple sub-shells, as well as the dissipation in the interaction between sub-shells and its effect on the outflow acceleration and the resulting emission, such as a possible photospheric spectral component.

\section{Dissipation and Prompt Emission}

As discussed above, GRBs must be associated with relativistic outflows ejected by a stellar mass compact source, with a bulk Lorentz factor $\Gamma \gtrsim 100$ in order to avoid the compactness problem (Baring and Harding 1997; Lithwick and Sari 2001; Granot et al. 2008; Hascoët et al. 2012). This also naturally explains the afterglow through the deceleration of the ejecta by the external medium, whereas the observed fast prompt variability implies that the prompt emission must be produced by internal dissipation within the ejecta (Sari and Piran 1997). Therefore, the analysis of the GRB prompt emission may provide valuable information on magnetic fields within an ultra-relativistic jet. It can put unique constraints on the state of the jet at the end of the acceleration phase, and more specifically on the geometry of the magnetic field and the magnetization at a large distance to the central source, where the $\gamma$-ray emission is produced. This is, however, a difficult task as it requires a full understanding of the nature of the dissipative mechanisms and of the radiative processes at work.

There are several possible emission sites for the GRB prompt emission: (i) a component can be emitted at the photosphere, where the ejecta becomes transparent to its own photons; another component can be produced above the photosphere in the optically thin regime, associated with either (ii) internal shocks propagating within the ejecta (Rees and Meszaros 1994); or (iii) magnetic reconnection (Thompson 1994, Spruit et al. 2001). These three dissipative mechanisms extract energy, respectively, from the thermal, kinetic or magnetic reservoirs. The expected prompt emission components are therefore strongly related to the composition of the ejecta. 


\subsection{Photospheric Emission}

\subsubsection{Non-Dissipative Photospheres}

When internal dissipation below the photosphere is negligible, the expected thermal emission at the photosphere is well understood with precise predictions (Paczynski 1986, Goodman 1986, Shemi and Piran 1990, Meszaros et al. 1993). Only few theoretical uncertainties remain, mainly related to the lateral structure of the jet (see e.g. Lundman et al. 2013; Deng and Zhang 2014). Assuming that the photosphere is above the saturation radius, the photospheric radius is given by (Mészáros and Rees 2000; Mészáros et al. 2002, Daigne and Mochkovitch 2002, Hascoët et al. 2013)

$$
R_{\mathrm{ph}} \simeq \frac{\kappa \dot{E}}{8 \pi c^{3} \Gamma^{3}(1+\sigma)} \simeq 3 \times 10^{13} \frac{\kappa_{0.2} \dot{E}_{53}}{\Gamma_{2}^{3}(1+\sigma)} \mathrm{cm},
$$

where $\kappa_{0.2}$ is the matter opacity in units of $0.2 \mathrm{~cm}^{2} \mathrm{~g}^{-1}, \Gamma=100 \Gamma_{2}$ is its bulk Lorentz factor, $\dot{E}=10^{53} \dot{E}_{53} \mathrm{erg} \mathrm{s}^{-1}$ is the isotropic equivalent jet power, and $\sigma$ is the magnetization parameter at the end of the acceleration, so that $\dot{E} /(1+\sigma)$ is the isotropic equivalent kinetic power. The observed photospheric luminosity and temperature are

$$
L_{\mathrm{ph}} \simeq \varepsilon_{\mathrm{th}} \dot{E}\left(\frac{R_{\mathrm{ph}}}{\Gamma R_{0}}\right)^{-2 / 3} \quad \text { and } \quad T_{\mathrm{ph}} \simeq \frac{T_{0}}{1+z}\left(\frac{R_{\mathrm{ph}}}{\Gamma R_{0}}\right)^{-2 / 3},
$$

where the initial temperature equals $T_{0}=\left(\varepsilon_{\mathrm{th}} \dot{E} / 4 \pi \sigma R_{0}^{2}\right)^{1 / 4}$. Here $\varepsilon_{\mathrm{th}}$ is the thermal fraction of the jet power at the base of the flow, located at the initial radius $R_{0}$, and $1-\varepsilon_{\mathrm{th}}$ is therefore the initial magnetic fraction. In the case of a passive magnetic field carried by the outflow without contributing to the acceleration, the initial thermal fraction $\varepsilon_{\text {th }}$ and the magnetization at the photosphere $\sigma$ are related by $\sigma_{\text {passive }}=\sigma_{0}=$ $\left(1-\varepsilon_{\mathrm{th}}\right) / \varepsilon_{\mathrm{th}}$. An efficient magnetic acceleration leads to $\sigma<\sigma_{\text {passive }}$ (Spruit et al. 2001). The predicted spectrum is quasi-thermal, with an exponential cutoff at highenergy and a power law at low-energy with a photon-index of $\alpha \simeq 0.4$, which differs from the $\alpha=1$ slope of the Raleigh-Jeans spectrum due to the peculiar geometric shape of a relativistic photosphere (Goodman 1986; Beloborodov 2011).

\subsubsection{Dissipative Photospheres}

If dissipation occurs below the photosphere, the emitted spectrum can be significantly different than the previous case: a high-energy tail can be produced by comptonization due to the presence of relativistic electrons (Thompson 1994; Mészáros and Rees 2000; Rees and Mészáros 2005, Giannios and Spruit 2007; Beloborodov 2010), and the low-energy slope can be modified by synchrotron radiation (Pe'er et al. 2006, Vurm et al. 2011). The resulting observed spectrum may now appear non-thermal, with several components. This scenario is more uncertain than the previous one. The nature of the sub-photospheric dissipative mechanism must be identified, with several candidates: early internal shocks, gradual magnetic reconnection (Thompson 1994 Giannios and Spruit 2007), neutron-proton collisions (Beloborodov 2010), etc. An important relevant issue is related to the photon production efficiency and thermalization deep within the ejecta (Vurm et al.2013). 
4.2 Non-Thermal Emission in the Optically Thin Regime

\subsubsection{Electron Acceleration and Synchrotron Radiation}

Non-thermal emission can be produced above the photosphere if some internal dissipation processes can lead to efficient electron acceleration. In this case, two natural candidates for the dominant radiative process are the synchrotron radiation and the inverse Compton scatterings of synchrotron photons by relativistic electrons (SSC). However, the measurement of the prompt $\gamma$-ray spectrum over a broad spectral range (keV-GeV) in a few bursts by Fermi/GBM+LAT can rule out the possibility of SSC being dominant in the soft $\gamma$-ray range, as it would lead either to a strong synchrotron peak at lower energy, or a strong second inverse Compton peak at higher energy, which are not observed (Bošnjak et al. 2009, Piran et al. 2009). Therefore, the discussion is focussed on the synchrotron radiation of relativistic electrons, with several possible dissipation mechanisms responsible for the acceleration of electrons.

\subsubsection{Internal Shocks}

If the magnetization at a large distance to the central source is sufficiently low, strong internal shocks are expected to form and propagate within the ejecta due to the variability of the ejected outflow. A large range of radii is expected,

$$
R_{\mathrm{is}} \simeq 2 \Gamma^{2} c\left(t_{\mathrm{var}} \rightarrow t_{\mathrm{GRB}}\right) \simeq \Gamma_{2}^{2}\left(6 \times 10^{12} t_{\mathrm{var},-2} \rightarrow 6 \times 10^{15} t_{\mathrm{GRB}, 1}\right) \mathrm{cm},
$$

where $t_{\mathrm{var},-2}$ is the shortest timescale of variability, in units of $10^{-2} \mathrm{~s}$ and $t_{\mathrm{GRB}, 1}$ is the total duration of the relativistic ejection, in units of $10 \mathrm{~s}$. The shocks are expected to be mildly relativistic, except for a very large amplitude of variation of the initial Lorentz factor. The dynamics of the internal shocks phase has been studied in detail (Kobayashi et al.|1997; Daigne and Mochkovitch 1998, 2000), from a simple ballistic approximation to a full hydrodynamical code, and is well understood. Up to $f_{\mathrm{d}} \simeq 40 \%$ of the kinetic energy can be dissipated (for a low magnetization outflow, $\sigma \ll 1$ ), depending again on the initial distribution of the Lorentz factor.

On the other hand, large uncertainties on the emission remain, due to the poor understanding of the microphysics of mildly relativistic shocks (for a recent review of relativistic collisionless shocks see Sironi et al. 2015). It is usually parametrized by assuming that a fraction $\varepsilon_{\mathrm{B}}$ of the internal energy is injected into an amplified random magnetic field at the shock, whose structure is not known, and a fraction $\varepsilon_{\mathrm{e}}$ is injected into a fraction $\zeta$ of electrons, which are therefore accelerated into a nonthermal distribution with slope $p\left(d N_{e} / d \gamma_{e} \propto \gamma_{e}^{-p}\right)$. To reach the soft $\gamma$-ray domain by synchrotron radiation, the fraction of accelerated electrons must be low, $\zeta \lesssim 10^{-2}$ (Daigne and Mochkovitch 1998, Bošnjak et al. 2009;, Daigne et al. 2011; Beniamini and Piran 2013). On the other hand, values of $\varepsilon_{\mathrm{e}}$ close to equipartition $\left(\varepsilon_{\mathrm{e}}=1 / 3\right)$ are required to explain the huge luminosities of GRBs. If the radiative efficiency is high, a fraction $f_{\mathrm{IS}} \simeq f_{\mathrm{d}} \varepsilon_{\mathrm{e}} \simeq 0.01-0.1$ of the initial kinetic power can be converted into radiation in internal shocks. With such assumptions, the non-thermal emission in the comoving frame of the shocked regions can be computed with a detailed radiative 
model including all the relevant processes, namely synchrotron radiation and slefabsorption, inverse Compton scatterings and photon-photon annihilation. The contributions of each internal shock can then be added with an integration over equal-arrival time surface of photons to the observer in the source frame to produce synthetic light curves and spectra that can be directly compared to observations. The predicted spectrum shows several components, a strong synchrotron peak in the soft $\gamma$-ray range and a weaker inverse Compton peak at higher energy (Bošnjak et al.2009).

\subsubsection{Magnetic Reconnection}

If the magnetization at large distances remains high $(\sigma \gtrsim 1)$, then internal shocks are either significantly suppressed or in some cases cannot form altogether (Mimica and Aloy 2010, Narayan et al. 2011). In such cases, electrons may be accelerated predominantly in magnetic reconnection sites. This scenario is even more uncertain (less understood) than the two previous ones (photosphere, internal shocks), but it is under extensive investigation (for a recent review on relativistic magnetic reconnection see Kagan et al. 2015). Some authors considered a gradual reconnection starting at a small radius and extending up to $R_{\text {rec }} \sim 10^{13} \mathrm{~cm}$ (Drenkhahn and Spruit|2002: Giannios 2008). In this case, most of the dissipation occurs below the photosphere, corresponding to the dissipative photosphere scenario discussed above. If, on the other hand, reconnection remains inefficient below the photosphere, it could occur at larger radii in the optically thin regime. In the simulations by McKinney and Uzdensky (2012), a catastrophic dissipation of the magnetic field occurs at $R_{\mathrm{rec}} \simeq 10^{13}-10^{14} \mathrm{~cm}$ when reconnection enters a rapid collisionless mode. Zhang and Yan (2011) proposed another scenario where reconnection is triggered by internal shocks, the socalled ICMART model. The typical radius may be as large as $R_{\mathrm{rec}} \simeq 10^{15} \mathrm{~cm}$. The microphysics in the reconnection sites is also uncertain. One expects many electron acceleration sites, which may move relativistically in the outflow's rest frame. The non-thermal electron distribution may be somewhat harder than in shock acceleration (see e.g. Sironi and Spitkovsky 2014; Kagan et al. 2015), and the acceleration process slower, which can lead to a different shape for the synchrotron emission. Contrary to internal shocks, detailed calculations of the light curves and spectra based on a detailed radiative model coupled to a dynamical simulation are not yet available.

\subsection{Magnetic Field in Emission Sites}

The models discussed above have very different implications for the magnetic field:

- Case 1: most of the prompt emission is due to a dissipative photosphere. Then, the magnetic field must be large enough at the photosphere to produce synchrotron radiation and affect the low-energy spectrum. If this magnetic field is generated by the dissipation process (e.g. shocks; Sironi et al. 2015), it is most probably random. Otherwise, an ordered field must be present. The corresponding initial magnetization must either be low (otherwise the photospheric emission is weak), or high with very efficient reconnection below the photosphere, which then leads to a possible candidate for the sub-photospheric dissipation process. 
- Case 2: the prompt emission is mostly non-thermal, from an optically thin region: - For internal shocks to be the dominant dissipation process, the magnetization at large distances from the source must be low. A random field is generated locally at the shock front, where the electrons are accelerated. However, the magnetic field felt by the radiating electrons must be considered far behind the shock front (as the radiative cooling length is much larger than the plasma skin depth) where its strength and structure are not well known.

- If reconnection dominates the dissipation then $\sigma$ must be large far from the source. The ordered field is destroyed at the reconnection sites, but if electrons have enough time to migrate from their acceleration site before radiating, their emission may still be mostly in the large-scale ordered field.

Observations of the GRB prompt emission, discussed in the next subsection, can put strong constraints on these various emission models.

\subsection{Constraints from the Observed Prompt Soft $\gamma$-ray Emission}

\subsubsection{Light Curves}

All the scenarios discussed above can reproduce the observed variable light curves. There are, however, important differences:

- (Dissipative) photosphere: the emission radius is low (see Eq. 15). Therefore the curvature effect, i.e. the spreading of photon arrival times from different angles with respect to the line of sight over an angular timescale $t_{\theta}=R / 2 \Gamma^{2} c$ of a flash of photons emitted at the same time and radius, is negligible: the observed light curve directly traces the activity of the central engine.

- Internal shocks: the light curves trace the source activity (Kobayashi et al. 1997; Daigne and Mochkovitch 1998), but two effects now affect the observed pulse shapes: the curvature effect (due to a larger radius) dominates the pulse decay (Genet and Granot 2009, Willingale et al. 2010), and the radial or hydrodynamic timescale due to shock propagation, $t_{r}=\Delta R / 2 \Gamma^{2} c$, dominates the pulse rise and overall shape (Daigne and Mochkovitch 1998, 2003, Bošnjak and Daigne 2014).

- Reconnection: again, the light curve traces the source activity, with new effects due to relativistic bulk motion in the local jet's frame. Relativistic motions of emitting plasma in the jet's frame cause rapid variability (that should show up as a distinct component in the Fourier power spectrum), while a slower envelope may arise from their combined effect (Zhang and Zhang|2014) or from slower emitting plasma. This can be tested by characterizing the observed variability. Analysis of GRB light curves shows a continuum of timescales (see e.g. Beloborodov et al. 2000; Guidorzi et al. 2012), which does not support the reconnection model of Zhang and Zhang (2014) (see however Gao et al. 2012). A possible concern appears if the emission is produced by many relativistically moving emitters: the predicted pulse shape may be too symmetric compared to observations (Lazar et al. 2009). However, both concerns (the power spectrum and pulse shapes) may be solved if the reconnection occurs in relatively ordered thin layers located between anti-parallel regions in the outflow (with a geometry of thin quasi-spherical 
shells) and the relativistic motions in the jet's frame are limited to these layers (Beniamini \& Granot 2015, in prep.). Such a model may also account for many of the correlations that are observed in the prompt emission.

\subsubsection{Polarization}

Measuring the polarization in the $\gamma$-ray domain remains challenging. A very large degree of polarization was claimed by Coburn and Boggs (2003), but it was later refuted by others (Rutledge and Fox 2004, Wigger et al. 2004) as not being statistically significant. Only a few later measurements (by INTEGRAL, GAP) are available, however with a low or moderate significance (McGlynn et al. 2007) Götz et al. 2009; Yonetoku et al. 2011, 2012; Götz et al. 2014). Such measurements (if reliable) can put constraints on the magnetic field geometry in the emission sites (e.g., Granot and Königl 2003, Granot 2003; Lyutikov et al. 2003, Nakar et al.|2003). Current observations seem to favor synchrotron radiation in an ordered field with patches, which would favor emission in the optically thin regime above the photosphere. It is however not trivial to justify a highly ordered field in the internal shocks model (a turbulent field at the shock is required for particle acceleration; The structure of the field on intermediate scales between the plasma and the dynamical scales is less known), or in the reconnection model (in principle the structured field is destroyed by reconnection, but the remaining field can still possess significant structure and electrons may also radiate somewhat outside of these localized reconnection regions). Either way, more definitive polarization observations are needed before strong conclusions can be drawn.

\subsubsection{Spectrum}

The prompt soft $\gamma$-ray spectrum is usually fitted by a phenomenological model introduced by Band et al. (1993), which consists of two power laws with low- and high-energy photon indices of $\alpha$ and $\beta$, smoothly connected at the peak energy $E_{\mathrm{p}}$. This eliminates non-dissipative photospheres for the dominant contribution to the emission, which puts a strong constraint on the initial magnetization (low $\varepsilon_{\mathrm{th}}$, Daigne and Mochkovitch 2002), favoring magnetic acceleration of the outflow. The remaining scenarios for the prompt GRB emission are either a dissipative photosphere, or a combination of a weak photospheric emission and a non-thermal component due to shocks or reconnection. The discussion is then focussed on the general shape of the spectrum, and the low-energy photon index $\alpha$, which is observed to be close to $\alpha \simeq-1$ (Preece et al. 2000, Kaneko et al.|2006; Nava et al. 2011; Gruber et al. 2014).

- Dissipative photospheres: the value of $\alpha$ can be reproduced by adjusting the magnetization, which controls the synchrotron emission at low energies. The theoretical instantaneous spectral peak is narrower than the observed time-integrated spectral peak, but the comparison should be made using a theoretical time-integrated spectrum, which should broaden it.

- Internal shocks: to reproduce the high luminosities and the short timescale variability of GRBs, the radiating electrons must be in the fast cooling regime (Cohen 
et al. 1997; Sari et al. 1998, Ghisellini et al. 2000), i.e. their radiative timescale must be shorter than the dynamical timescale that governs the adiabatic cooling for the spherical expansion. This leads to a predicted photon index $\alpha \leq-3 / 2$, in contradiction with observations (the so-called "synchrotron line-of-death", Preece et al. 1998). Another potential problem is that the resulting spectrum is too broad around the peak. The two problems are naturally connected. Several possibilities have been discussed to solve this issue: (i) inverse Compton scatterings in the Klein-Nishina regime affect the cooling of electrons, leading to photon indices $\alpha \lesssim-1$ (Derishev et al. 2001; Wang et al. 2009; Nakar et al. 2009; Daigne et al. 2011). This puts a constraint on the strength of the magnetic field, which should be small, with $\varepsilon_{\mathrm{B}} \lesssim 10^{-3}$ (Daigne et al. 2011; Barniol Duran et al. 2012); (ii) in the marginally fast cooling regime (Daigne et al. 2011; Beniamini and Piran 2013), where the radiative timescale is close to the dynamical timescale but still below, the electron radiative efficiency can remain large enough $(\gtrsim 50 \%)$ to explain the observed luminosities, but the synchrotron spectrum is strongly affected: the intermediate region of the spectrum below the peak with a photon index $-3 / 2$ disappears and the slope $\alpha=-2 / 3$ usually associated with the inefficient slow cooling regime is measured. This regime is also favored by weak magnetic fields; (iii) in the fast cooling regime, electrons radiate on timescales which are long compared to the plasma scale at the shock front, but small compared to the dynamical timescale. Then they experience a magnetic field that is not necessarily the same as the turbulent field just behind the shock (see the structure of the magnetic field in the simulations, e.g. Keshet et al. 2009). If the field is decaying on this intermediate scale, it will affect the synchrotron spectrum and can lead to a hard spectrum, with $-1 \lesssim \alpha \lesssim-2 / 3$ (Derishev 2007; Lemoine 2013, Uhm and Zhang 2014; Zhao et al. 2014).

- Reconnection: the slow electron heating in the turbulent field can lead to hard synchrotron spectra with $\alpha \simeq-1$ (Uhm and Zhang 2014). It is unclear if the expected hard power-law index $p \lesssim 1.5$ of the non-thermal electron distribution (e.g., Sironi and Spitkovsky 2014) can be identified in the observed spectrum. A potential issue is that the presence of many emitting regions that move relativistically in random directions in the jet's fame might lead to much broader spectra than observed. This may be alleviated, however, in models where these regions move predominantly along the thin reconnection layer that is located between regions of oppositely-directed magnetic field in the flow, normal to the jet's bulk motion (Beniamini \& Granot 2015, in prep.).

Recently the description of observed GRB spectra in the soft $\gamma$-ray range has been greatly improved by Fermi/GBM observations. An important result is the identification of significant deviations from the Band spectrum, which seem to be related to the presence of a weak thermal component? below the dominant non-thermal one (see e.g. Ryde et al. 2010, 2011; Guiriec et al. 2011; Burgess et al. 2011; Axelsson et al. 2012; Guiriec et al. 2013; Burgess et al. 2014, Guiriec et al. 2015), as illustrated in Figure 7. A natural explanation is to associate the quasi-thermal weak component to a (non-dissipative) photosphere and the Band component to synchrotron radiation

\footnotetext{
${ }^{9}$ Or possibly even a dominant photospheric component in the case of GRB 090902B.
} 

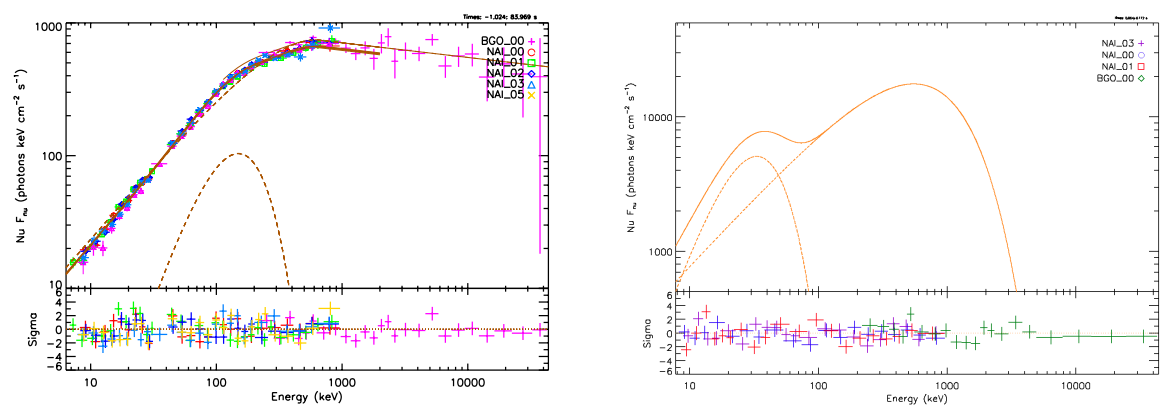

Fig. 7 Two examples of quasi-thermal components detected in GRB prompt spectra. Left: a weak quasithermal component at $T \simeq 38 \mathrm{keV}$ in the long GRB 100724B ( from Guiriec et al. 2011). Right: a quasithermal component at $T \simeq 12 \mathrm{keV}$ in the short GRB 120323A (from Guiriec et al. 2013).
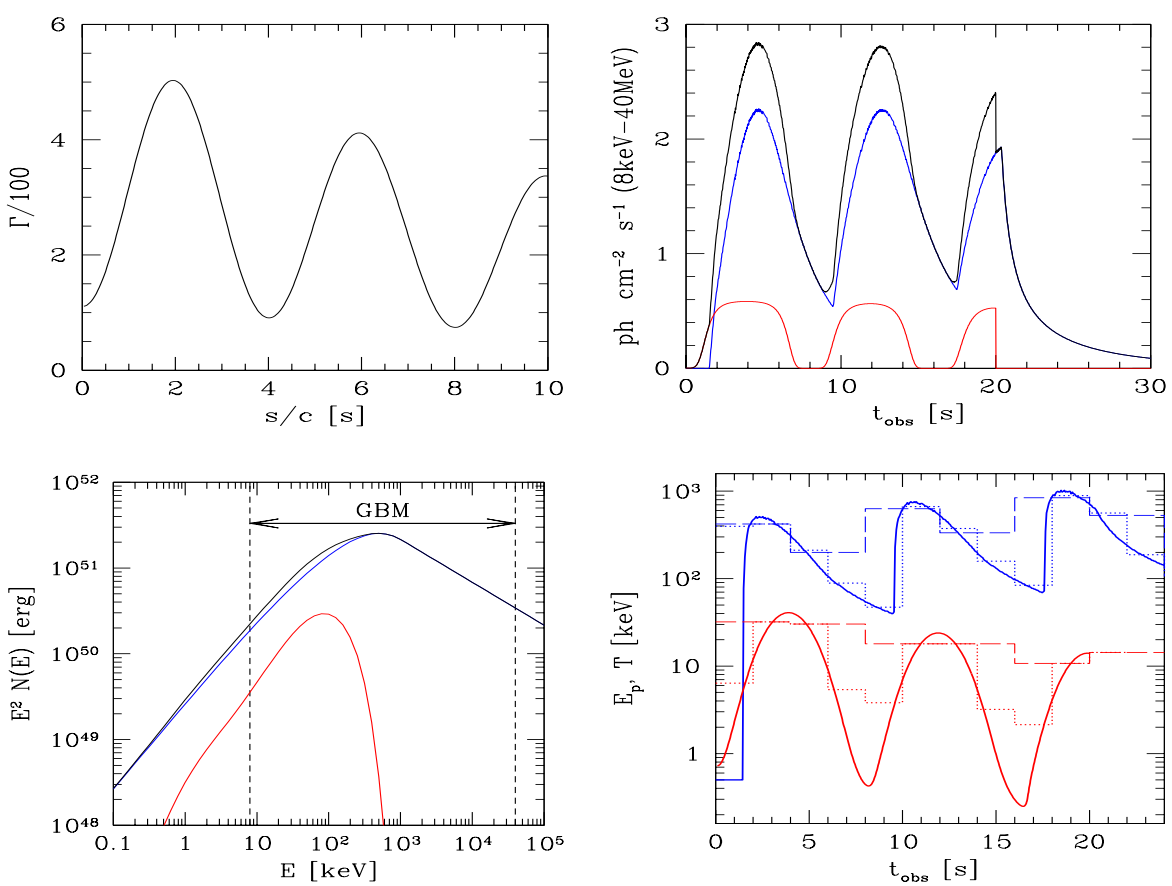

Fig. 8 An example of a synthetic GRB with the contribution from the photosphere and internal shocks computed self-consistently. The photospheric emission is plotted in red, the non-thermal emission from internal shocks in blue, the total in black. Top-left: initial distribution of the outflow Lorentz factor at the end of the acceleration phase. Top-right: light-curves in the GBM energy range. Bottom-left: spectrum. Bottom-right: spectral evolution (observed peak energy of the non-thermal component and temperature of the photosphere). The dashed and dotted lines show the expected result when integrating the spectrum over different timescales. The parameters are $\dot{E}=10^{53} \mathrm{erg} / \mathrm{s}, \varepsilon_{\mathrm{th}}=0.03$ (high initial magnetization, $\sigma_{0}=32.3$ ), $\sigma=0.1$ (low magnetization at large distance), $R_{0}=3 \times 10^{7} \mathrm{~cm}$. (All panels are taken from Hascoët et al. 2013 . 
from electrons accelerated either in shocks or in reconnection: Figure 8 shows an example of a synthetic burst with these two contributions in the case of internal shocks. The weakness of the photospheric emission puts interesting constraints on the initial magnetization of the outflow (Daigne and Mochkovitch 2002, Hascoët et al. 2013), favoring an efficient magnetic acceleration, with a large range of initial magnetization in the GRB population, $\varepsilon_{\mathrm{th}} \lesssim 0.01\left(\sigma_{0} \gtrsim 100\right)$ in most cases where no detection is made and $\varepsilon_{\mathrm{th}} \simeq 0.01-0.1\left(\sigma_{0} \simeq 10-100\right)$ in less frequent cases like GRB 100724B (Hascoët et al. 2013). GRB 090902B with $\varepsilon_{\text {th }} \simeq 0.3-1\left(\sigma_{0} \lesssim 2.3\right)$ remains an exception within long GRBs, and the short GRB 120323A appears as an intermediate case between GRBs 100724B and 090902B with $\varepsilon_{\text {th }} \simeq 0.1-0.5\left(\sigma_{0} \simeq 1-9\right)$ (Guiriec et al. 2013). The fact that the photospheric emission seems brighter in the only case of detection in a short GRB (GRB 120323A, Guiriec et al. 2013) may indicate a different acceleration mechanism. If this interpretation is correct, these recent detections rule out purely thermal acceleration (standard fireball) at least in long GRBs.

\subsection{Constraints on a Poynting Flux Dominated Outflow}

Several authors (Lyutikov 2006; Giannios and Spruit 2006; Zhang and Yan 2011) have proposed that GRB jets are Poynting flux dominated all the way up to the emission region. The prompt $\gamma$-rays arise, in this case, from a process that converts this magnetic energy to radiation. Obviously, this cannot take place directly and one has to invoke some sort of magnetic dissipation (e.g. reconnection) that converts the magnetic energy to accelerated electrons (or electron-positron pairs) that emit the observed $\gamma$-rays. Particular support for this idea came with the claim of of strong polarization in the prompt emission by Coburn and Boggs (2003), which was later refuted (Rutledge and Fox 2004; Wigger et al. 2004). Such polarization could arise if the magnetic field is ordered and this will arise naturally if the magnetic field is dominant (Granot and Königl 2003; Granot 2003; Lyutikov et al. 2003).

However, the efficiency of the synchrotron emission process poses serious constraints on models in which the emission region is Poynting flux dominated (Beniamini and Piran 2014). Consider a Poynting flux dominated outflow and an observed (isotropic equivalent) $\gamma$-ray luminosity $L_{\gamma}$. This luminosity immediately sets a lower limit on the strength of the magnetic field $B$ in the rest frame ${ }^{10}$ of the central source, $L_{\gamma}<R_{\mathrm{em}}^{2} B^{2} c$, where $R_{\mathrm{em}}$ is the emission radius. Accelerated electrons effectively emit synchrotron radiation. The critical issue here is that synchrotron emission is too efficient. The accelerated electrons cool so rapidly in a strong magnetic field that their lower bands (X-rays and optical) synchrotron emission would produce a signal that is much stronger than the observed emission in these bands.

The observed prompt upper limits in the optical or the X-rays set strong constraints on the conditions within the emitting region. First, if the observed $\gamma$-rays are due to some other (non-synchrotron) emission process then this process must be extremely efficient and its cooling time should be significantly shorter than the relevant synchrotron cooling time (see e.g. Figure 9).

10 The magnetic field in the jet's frame is $B^{\prime}=B / \Gamma$, where $\Gamma$ is its bulk Lorentz factor. 


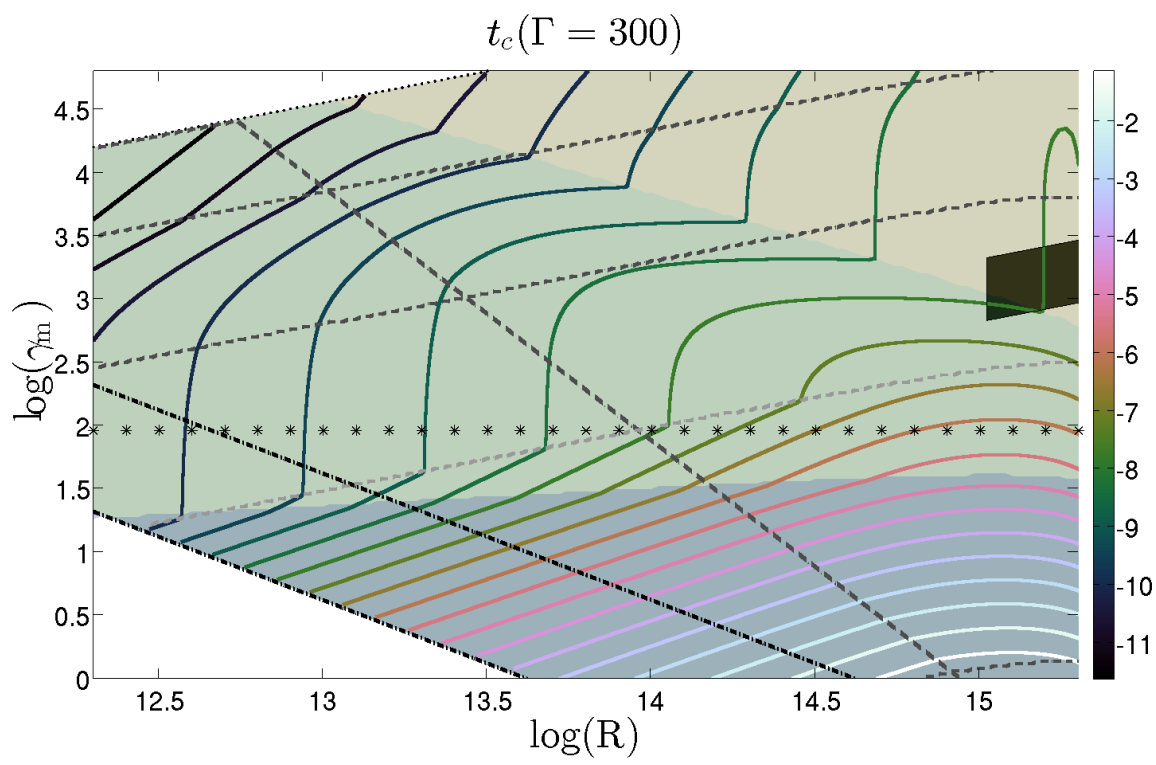

Fig. 9 If the dominant $\gamma$-ray emission mechanism is not synchrotron, then in order for it to be able to tap a significant fraction of the electrons' energy its cooling time, $t_{\mathrm{c}}$, must be shorter than that due to synchrotron radiation, $t_{\mathrm{c}, \mathrm{syn}}$, which is depicted here by the contour lines. The observational constraints $F_{v \text {,syn,opt }}<1 \mathrm{mJy}, F_{v \text {,syn,X-ray }}<1 \mathrm{mJy}$ and $v_{\text {syn,LAT }} F_{v, \text { syn,LAT }}<10^{-7} \mathrm{erg} \mathrm{s}^{-1} \mathrm{~cm}^{-2}$ further constrain $t_{\mathrm{c}}$. Beyond the corresponding lines, $t_{\mathrm{c}}$ should be significantly shorter than $t_{\mathrm{c}, \text { syn }}$ in order for the synchrotron not to overproduce the upper limits on the optical, $\mathrm{X}$-ray or $\mathrm{GeV}$ fluxes. Within the black region the synchrotron emission produces the observed prompt $\gamma$-rays. The conditions $\tau_{T}<1,10$ (dot-dashed lines; a Thompson optical depth that is not too large) define general limits on the parameter space. (see Beniamini and Piran 2014 for more details).

Alternatively, if the observed prompt $\gamma$-ray emission is synchrotron then there must be a rapid reaccelerating process that keeps the electrons with the right Lorentz factor so that they would not cool too much and emit strongly in lower energy bands, in particular in soft X-rays (Ghisellini and Celotti 1999, Kumar and McMahon 2008, Fan 2010). This requires strong fine tuning as the Lorentz factor range in which the electrons must be kept is rather narrow (a factor of $\sim 3-10$ ). Multi-zone configurations in which the electrons escape the emitting region before cooling and overproducing X-ray or optical emission are also a possibility (e.g. as in the ICMRAT model Zhang and Yan 2011). Beniamini and Piran (2014) considered several such two-zone toy models (in which electrons are accelerated in one region and emit in the other) but proper conditions could not be found in any of them.

These considerations pose severe constraints on prompt emission models that involve Poynting flux dominated outflows. Any emission model in such a regime should satisfy these constraints. Lacking a model that satisfies all these constraints, it is likely that if the outflow is initially Poynting flux dominated then the magnetic energy is dissipated before the emitting region, where it must be subdominant. 
4.6 Spectral Diversity - Spectral Evolution

The peak energy $E_{\mathrm{p}}$ varies a lot from one GRB to another, from a few to tens of keV (X-ray Flashes, X-ray Rich GRBs; Sakamoto et al.2005) to over $10 \mathrm{MeV}$ (Axelsson et al. 2012). An important property is that short GRBs are harder with larger peak energies (Kouveliotou et al. 1993; Guiriec et al. 2010). Spectral evolution is also found: when time-resolved spectroscopy is possible, the GRB spectrum is always found to strongly evolve during the prompt phase (see e.g. Lu et al. 2012, Burgess et al. 2014, Guiriec et al. 2015 for recent analyses of Fermi GRBs, or Preece et al. 2014 for a very bright case where the spectral evolution can be studied in great detail); $E_{\mathrm{p}}$ typically varies over more than a factor of 30 within an individual pulse. Spectral and temporal properties appear correlated within GRB pulses: hardness following the intensity, pulses being narrower and peaking earlier at higher energies, etc.

Both the spectral diversity between different GRBs and the spectral evolution within individual GRBs are hard to reproduce by models. Reconnection models are barely developed enough to allow discussion of these observations. In dissipative photospheres, variations in $E_{\mathrm{p}}$ are related to changes in the properties of the outflow ejection leading to a change in the location of the photosphere (Pe'er 2008; $\mathrm{Be}$ loborodov 2013; Deng and Zhang 2014). A potential issue is to explain how the dissipative process adjusts to always remain located just below the photosphere (unless it always occurs over a wide range of radii, in which case it should also occur above the photosphere, so this would not be a pure photospheric model). In internal shocks, the spectral evolution is reproduced qualitatively (Daigne and Mochkovitch 1998 2003; Asano and Mészáros 2011, Bošnjak and Daigne 2014), and can even be reproduced quantitatively with some constraints on microphysics parameters (Bošnjak and Daigne 2014), which may indicate non-universal values in mildly relativistic shocks, as suggested for instance by Bykov and Meszaros (1996, see also Bykov et al. 2012). The spectral diversity is also naturally explained by variations in the lifetime and variability of the central engine (Barraud et al. 2005). The hardness-duration relation is well reproduced (Daigne and Mochkovitch 1998; Bošnjak and Daigne 2014).

\subsection{Constraints at Other Wavelengths}

The discussion above was centered on observations in the soft $\gamma$-ray range, where the prompt emission is observed in most GRBs. We discuss here briefly some additional constraints coming from observations of the prompt emission at other wavelengths.

\subsubsection{The End of the Prompt Emission: the X-ray Early Steep Decay}

Swift/XRT discovered in most GRB X-ray afterglows an early steep decay at the end of the prompt phase, before recovering a plateau and/or a standard afterglow decay (Nousek et al. 2006, Zhang et al. 2006, O'Brien et al.|2006). A natural explanation is provided by the high-latitude tail of the prompt emission, once the on-axis emission has stopped (Kumar and Panaitescu 2000, Genet and Granot 2009). It can reproduce the observed temporal decay and spectral evolution (Liang et al. 2006, Willingale 
et al. 2010). This puts a strong constraint on the emission radius at the end of the prompt phase, which may be fulfilled by internal shocks, and also possibly by reconnection models if the radius is large enough (Hascoët et al.2012). On the other hand, it is incompatible with photospheric models, which must instead explain the rapid decay phase by a universal behavior of the central engine when it is switching off.

\subsubsection{Prompt GeV Emission}

Fermi/LAT detects GeV emission in some GRBs (Ackermann et al. 2013). As detection requires enough photons in its energy range (tens of $\mathrm{MeV}$ to $\gtrsim 300 \mathrm{GeV}$ ), it detects mainly very bright GRBs, in terms of both their GeV fluence and their total fluence (and thus also in terms of $E_{\gamma, \text { iso }}$ ). For the same reason, LAT detects a smaller fraction of short GRBs compared to soft $\gamma$-ray instruments, since their fluence is typically much smaller than that of long GRBs. Bright enough LAT GRBs show a distinct high-energy spectral component, usually fitted by a power law (e.g., Abdo et al. 2009; Ackermann et al. 2010, 2013). The observed variability in the prompt LAT light curve indicates an internal origin. It is followed by a long-lasting emission (with a power law in time and energy) that likely originates from the deceleration phase or early afterglow. In dissipative photospheric models, it is hard to produce $\mathrm{GeV}$ photons due to strong $\gamma \gamma$ annihilation. However, additional processes such as later scatterings of prompt photons by the external medium can explain this $\mathrm{GeV}$ emission (see e.g. Beloborodov et al.|2014). In reconnection models, spectral models cannot make such predictions yet. In internal shocks, such multi-component spectra are expected (Guetta and Granot 2003; Bošnjak et al. 2009; Asano and Mészáros 2012; Bošnjak and Daigne 2014); the fact that the $\mathrm{GeV}$ component is usually weaker than the soft $\gamma$-ray component constrains the strength of the magnetic field, implying that it must be weak, $\varepsilon_{\mathrm{B}} \lesssim 10^{-2}$ (Daigne et al. 2011; Bošnjak and Daigne 2014).

\subsection{Prompt Emission Summary}

The dissipation mechanism and radiative processes responsible for the prompt GRB emission are still not well understood due to the complex physics involved, both on large and micro-scales. The lack of strong thermal components in GRB spectra suggests a high initial magnetization in GRB outflows, while prompt GRB observational constraints imply a low magnetization in the emission region. Put together, this strongly suggests either very efficient conversion of magnetic to kinetic energy, which leaves a low magnetization in the emission region, and allows for efficient internal shocks (Granot et al. 2011, Granot 2012b), or strong magnetic reconnection that converts magnetic energy to thermal energy and accelerates particles, and yet somehow leaves a low enough magnetization where these particle radiate most of their energy.

A weak thermal emission can be produced at the photosphere, followed by a dominant non-thermal emission at larger radii. Depending on the efficiency of the acceleration and the resulting magnetization at large distances, the dissipation leading to the emission can occur either in shocks or in magnetic reconnection. In both cases, the dominant radiative process should be synchrotron emission. Only in the first case 
(internal shocks), detailed simulations coupling a dynamical calculation with a detailed radiative model are available. To have a good agreement between the observed spectrum and the predicted one, detailed modeling is needed, where the strength and structure of the magnetic field play a crucial role: moderately efficient inverse Compton scatterings in the Klein-Nishina regime are needed, which requires a weak field, and a decay of the magnetic field far from the shock front is also probably required.

An alternative is to explain the whole soft $\gamma$-ray emission by a dissipative photosphere. Its nature, however, must then be elucidated. The magnetic field could again play a vital role, via the magnetic reconnection below the photosphere, which is a natural candidate. Further progress can come from more observations over a broad spectral range with time-resolved spectroscopy, additional and firmer polarization measurements, and improvements in the modeling of the expected spectrum and spectral evolution in each model. Unfortunately, it remains limited by the current knowledge of the microphysics (structure of the magnetic field, particle acceleration) in mildly relativistic shocks and magnetic reconnection (Sironi et al. 2015; Kagan et al. 2015).

\section{Magnetic Fields in the Afterglow}

Eventually, the GRB outflow is decelerated by the external medium. It drives a strong relativistic blast wave - the afterglow (or external forward) shock - into the surrounding medium. It transfers most of its energy to the shocked external medium (via $p d V$ work across the contact discontinuity that separates them) at a distance $R_{\mathrm{dec}}$ from the central source - the deceleration radius. Radiation from $R_{\text {dec }}$ reaches the observer at the deceleration time, $T_{\mathrm{dec}}$. At $R>R_{\mathrm{dec}}$ the original outflow composition no longer affects the dynamics (or emission) of the afterglow shock. However, the outflow magnetization can greatly affect the reverse shock (or external reverse shock, as it is formed due to the interaction with the external medium), whose strength and emission can be greatly suppressed if the outflow is highly magnetized, $\sigma\left(R_{\mathrm{dec}}\right) \gtrsim 1$.

\subsection{The Afterglow Emission}

The dominant emission mechanism in the afterglow is thought to be synchrotron radiation, which is produced by relativistic electrons accelerated at the afterglow shock that gyrate in the magnetic fields within the shocked external medium ${ }^{11}$ Such a synchrotron origin of the afterglow emission is strongly supported by the detection of linear polarization at the level of $\sim 1-3 \%$ in several optical or NIR afterglows (see $\$ 5.2$, and by the shape of the broadband spectrum, which consists of several power law segments that smoothly join at some typical break frequencies. Figure 10 shows the possible resulting afterglow spectra. Broadband (radio to $\gamma$-ray) afterglows fit such synchrotron spectra far better than the prompt emission. The broad and mostly

\footnotetext{
11 In an alternative scenario, the afterglow emission is dominated at early times by the contribution of a long-lived reverse shock (Uhm and Beloborodov 2007. Genet et al. 2007), which allows to reproduce more easily the observed diversity and variability, such as X-ray plateaus (Uhm et al.|2012, Hascoët et al.|2014) or X-ray flares (Hascoet et al.2015), though in this scenario a transition to forward shock dominance is expected at late times but not observed.
} 
featureless smoothly broken power-law shapes of afterglow spectra evolve and fade more slowly over time, and have characteristic frequencies that vary as a power law with time, roughly according to the theoretically expected power-law indices (Sari et al. 1998, Granot and Sari 2002). Synchrotron self-Compton (SSC) - the inverseCompton scattering of the synchrotron photons to (much) higher energies by the same population of relativistic electrons that emits the synchrotron photons - can sometimes dominate the afterglow flux in the X-rays (Sari and Esin 2001, Harrison et al. 2001), and may affect the synchrotron emission by increasing the electron cooling.

Relativistic collisionless shock physics (e.g., how they amplify the magnetic field and accelerate a non-thermal population of relativistic particles) are still not well understood from first principles (e.g. Sironi et al. 2015). Thus, simple assumptions are usually made that conveniently parameterize our ignorance. The electrons are assumed to be (instantly) shock-accelerated into a power-law distribution of energies, $d N / d \gamma_{e} \propto \gamma_{e}^{-p}$ for $\gamma_{e}>\gamma_{m}$, and then cool both adiabatically and due to radiative losses 12 The relativistic electrons are assumed to hold a fraction $\varepsilon_{e}$ of the internal energy immediately behind the shock, while the magnetic field is assumed to hold a fraction $\varepsilon_{B}$ of the internal energy everywhere in the shocked region. Both the temporal and spectral indices depend on the power law index $p$ of the electron energy distribution. The temporal index (i.e. the rate of flux decay) also depends on the circumburst density profile, which is parameterized in Figure 10 as a power law of index $k$ with the distance $R$ from the central source, $\rho_{\mathrm{ext}} \propto R^{-k}$, with $k=0$ and $k=2$, respectively, corresponding to an ISM and a stellar wind - WIND. The temporal index can also be affected by other factors, such as energy losses or injection into the afterglow shock, the afterglow jet angular structure and the viewing angle relative to the jet symmetry axis, or time evolution of the shock microphysics parameters $\varepsilon_{e}$ and/or $\varepsilon_{B}$.

\subsection{Polarization: Afterglow and Reverse Shock}

The detection of linear polarization of a few percent in the optical and NIR afterglow of several GRBs (see Covino et al. 2004, and references therein) was considered as a confirmation that synchrotron radiation is the dominant afterglow emission mechanism. The synchrotron emission from a fluid element with a locally uniform magnetic field is linearly polarized in the direction perpendicular to the projection of the magnetic field onto the plane normal to the wave vector. Since the source moves relativistically, one must account for aberration of light when calculating the observed local direction of polarization. Figure 11 shows the predicted local polarization map from emission by an ultra-relativistic expanding shell, for two different magnetic field structures: a magnetic field that is random within the plane normal to the radial direction (left panel) as could be expected from a shock-produced field (e.g., Medvedev and Loeb 1999), and an ordered magnetic field normal to the radial direction (right panel; as could be expected in the prompt or reverse-shock emission for a magnetic field coherent on angular scales $\gg 1 / \Gamma$ that is advected from the central source).

\footnotetext{
12 It is usually also further assumed that practically all of the electrons take part in this acceleration process and form such a non-thermal (power-law) distribution, leaving no thermal component (which is not at all clear or justified; e.g. Eichler and Waxman 2005).
} 




Fig. 10 The afterglow synchrotron spectrum, calculated for the Blandford and McKee (1976) spherical self-similar solution, under standard assumptions, using the accurate form of the synchrotron spectral emissivity and integration over the emission from the whole volume of shocked material behind the forward (afterglow) shock (for details see Granot and Sari 2002 from which this figure is taken). The different panels show the five possible broad band spectra of the afterglow synchrotron emission, each corresponding to a different ordering of the spectral break frequencies. Each spectrum consists of several power-law segments (PLSs; each shown with a different color and labeled by a different letter A-H) that smoothly join at the break frequencies (numbered 1-11). The broken power-law spectrum, which consists of the asymptotic PLSs that abruptly join at the break frequencies (and is widely used in the literature), is shown for comparison. Most PLSs appear in more than one of the five different broad band spectra. Indicated next to the arrows are the temporal scaling of the break frequencies and the flux density at the different PLSs, for a uniform (ISM; $k=0$ ) and stellar wind (WIND; $k=2$ ) external density profile. 

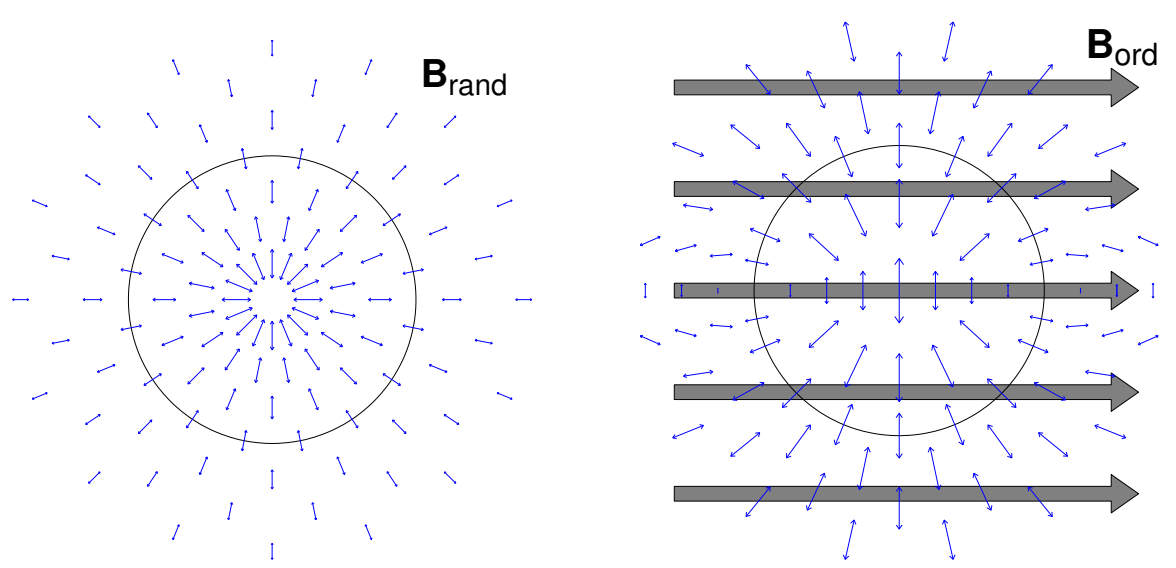

Fig. 11 The predicted polarization map for synchrotron emission from a thin spherical ultra-relativistic shell expanding with a Lorentz factor $\Gamma \gg 1$. The double-sided arrows show the direction of the linear polarization (the wave electric vector), while their length depends monotonically on the polarized intensity (in a non-trivial way, for display purposes). The circle indicates an angle of $1 / \Gamma$ around the line of sight to the central source, and contains the region responsible for most of the observed flux. Left: for a magnetic field that is random within the plane of the shell (normal to the radial direction), for which the polarization direction always points at the center of the image, where the polarization vanishes (due to symmetry consideration). Right: for an ordered magnetic field within the plane of the shell that is coherent over angular scales $\gg 1 / \Gamma$ (Granot and Königl 2003). In this case the direction of the ordered magnetic field clearly breaks the symmetry around the center of the image, resulting in a large net polarization. For simplicity, the map is for a constant emission radius, rather than for a constant photon arrival time.

The afterglow image is almost always unresolved, so we can only measure the (weighted) average polarization over the whole image. Therefore, a shock produced magnetic field that is symmetric about the shock normal will procure no net polarization for a spherical flow (as in this case the polarization pattern across the image is symmetric around its center, and the polarization averages out to zero when summed over the the whole image). For a shock-produced magnetic field, one thus needs to break this symmetry of the emission to produce net polarization. A simple and natural way of doing this is considering a jet, or narrowly collimated outflow (e.g., Sari 1999, Ghisellini and Lazzati 1999). In this picture a jet geometry together with a line of sight that is not along the jet symmetry axis (but still within the jet aperture, in order to see the prompt GRB) is needed to break the symmetry of the afterglow image around our line of sight. Other models for afterglow polarization include a magnetic field that is coherent over patches of a size comparable to that of causally connected regions (Gruzinov and Waxman 1999), polarization that is induced by microlensing (Loeb and Perna 1998) or by scintillations in the radio (Medvedev and Loeb 1999), a small ordered magnetic field component originating from the circumburst medium (Granot and Königl|2003), clumps in the external medium (Granot and Königl 2003), or a very inhomogeneous jet angular structure - a patchy shell with hot spots (Granot and Königl 2003, Nakar and Oren 2004). The many possible causes of polarization, and the degeneracy with other factors makes it difficult to robustly determine the magnetic field structure in the emitting region from afterglow polarization measure- 
ments. Nonetheless, a high degree of linear polarization with a stable position angle is hard to produce without a magnetic field that is ordered on large scales.

The reverse shock has two main observational signatures: a sharply-peak "optical flash" (e.g., Akerlof et al. 1999) on a timescale comparable to the prompt GRB $T_{90}$, and a "radio flare" (e.g., Kulkarni et al. 1999, Frail et al. 2000, Berger et al. 2003) that peaks on a timescale of a day or so after the GRB. In such cases, if the relatively bright observed emission is indeed from the reverse shock, this implies that the outflow was not strongly magnetized near the deceleration epoch, $\sigma\left(R_{\mathrm{dec}}\right) \ll 1$. Moreover, the polarization properties of the synchrotron emission from the reverse shock provide a powerful and unique probe for the magnetic field structure in the original outflow. Early optical polarization measurements from $T \gtrsim T_{\mathrm{dec}}$ have finally been obtained in the last eight years or so (Mundell et al.2007a, 2013; Steele et al. 2009).

On the one hand, there is a strict upper limit on the degree of linear polarization from GRB 060418 of $P<8 \%(2-\sigma)$ at $T=203 \mathrm{~s}$ after the GRB trigger, while the deceleration time suggested by the early optical lightcurve of this GRB is $T_{\mathrm{dec}}=$ $153 \pm 10 \mathrm{~s}$ (Molinari et al.2007) and its prompt emission lasted only $T_{90}=52 \pm 1 \mathrm{~s}$. The fact that $T_{\mathrm{dec}} / T_{90} \approx 3$ suggest a "thin shell" in this case, which is consistent with a moerate magnetization $\left(\sigma\left(R_{\mathrm{dec}}\right) \ll 1\right)$ that allows a strong reverse shock with bright emission. However, the polarization is fairly low near $T_{\mathrm{dec}}=153 \pm 10 \mathrm{~s}$ (at $T=203 \mathrm{~s}$ ), which suggests that either the reverse shock emission even near its peak is for some reason greatly sub-dominant compared to the (very weakly polarized) forward shock emission, or more likely that in this GRB there is hardly any ordered magnetic field in the ejecta on angular scales $\gtrsim 1 / \Gamma$ that cover most of the visible region.

On the other hand, GRB 090102 had a prompt duration of $T_{90}=27 \mathrm{~s}$ and an optical linear polarization of $P=10.2 \pm 1.3 \%$ in a $60 \mathrm{~s}$ exposure starting at $T=161 \mathrm{~s}$ after the trigger time (Steele et al. 2009). Its optical lightcurve shows a power-law decay $F_{v} \propto t^{-\alpha}$ with $\alpha=1.50 \pm 0.06$ from $T \sim 40 \mathrm{~s}$ to $T \sim 1000 \mathrm{~s}$ and then flattens to $\alpha=0.97 \pm 0.03$ (Gendre et al. 2010). This suggests a deceleration time $T_{\mathrm{dec}} \lesssim 40 \mathrm{~s}$, well before the polarization measurement. However, the optical emission may be dominated by the reverse shock up to the break time of $\sim 1000 \mathrm{~s}$, and in particular during the polarization measurement. In the latter case this might possibly explain the measured polarization as arising from an ordered magnetic field component in the ejecta, though a purely ordered field on the scale of the whole emitting region (angular scale $\gtrsim 1 / \Gamma$ ) would produce a significantly larger polarization of several tens of percent (Granot and Königl 2003, Granot 2003; Lyutikov et al. 2003, Nakar et al. 2003), which would suggest either a smaller magnetic field coherence length or a dominant contribution from the much less polarized external forward shock emission.

Finally, GRB $120308 \mathrm{~A}$ that lasted $T_{90} \sim 100 \mathrm{~s}$ (between $T \approx-30 \mathrm{~s}$ and $T \approx 70 \mathrm{~s}$ post-trigger) showed an optical linear polarization of $P=28 \pm 4 \%$ in an exposure between $T=240 \mathrm{~s}$ and $323 \mathrm{~s}$ (Mundell et al. 2013), which gradually decreased to $P=16_{-4}^{+5} \%$ over the next ten minutes, while keeping an approximately constant position angle (to within an accuracy of about $15^{\circ}$ ). The optical lightcurve peaked at around $T \sim 300 \mathrm{~s}$, during the time bin in which the largest polarization was measured, and subsequently decayed, with a possible transition from reverse to forward shock domination of the optical emission around $\sim 1000 \mathrm{~s}$. This strongly suggests the presence of a large-scale ordered magnetic field in the original GRB ejecta. 
Observations of radio flares at roughly a day after the GRB have so far produced no detection of polarization. However, these observations have enabled to set strict upper limits on a possible linear or circular polarization (Granot and Taylor 2005). The strictest limits are for GRB 991216, for which the $3-\sigma$ upper limits on the linear and circular polarization are $P_{\text {lin }}<7 \%$ and $P_{\text {circ }}<9 \%$, respectively. These limits provide interesting constraints on existing GRB models (Granot and Taylor 2005), and in particular are hard to reconcile with a predominantly ordered toroidal magnetic field in the GRB outflow together with a "structured" jet, where the energy per solid angle drops as the inverse square of the angle from the jet axis, as is expected in some models in which the outflow is Poynting flux dominated.

Recently, the detection of circular polarization was reported in the optical afterglow of GRB 121024A by Wiersema et al. (2014). In particular, they measured a circular polarization of $P_{\text {circ }}=0.61 \pm 0.13 \%$ at $T=0.15$ days after the GRB. The linear polarization during that time was $P_{\text {lin }} \sim 4 \%$ implying a circular to linear polarization ratio of $P_{\text {circ }} / P_{\text {in }} \sim 0.15$. A very recent detailed study that examined different assumptions for the magnetic field configuration, jet geometry and electron pitchangle distribution (Nava et al. 2015) concluded that such a relatively high $P_{\text {circ }} / P_{\text {lin }}$ ratio cannot be produced by synchrotron emission from the afterglow (i.e. forward external) shock, which suggests an alternative origin.

\subsection{Maximum Synchrotron Photon Energy}

Since the afterglow emission from the shocked external medium is independent of the outflow composition, it can much more "cleanly" probe the physics of relativistic collisionless shocks, and serve as a testbed for how the very weak upstream magnetic fields in the pristine surrounding medium are amplified in the shock, and how the particles are accelerated in this shock and radiate in the downstream magnetic field.

A recent challenge to the standard synchrotron afterglow scenario was raised by the exceptional GRB 130427A (Ackermann et al. 2014, Perley et al. 2014; Kouveliotou et al. 2013; Maselli et al. 2014). This was a very energetic GRB (with an isotropic equivalent $\gamma$-ray energy of $E_{\gamma, \text { iso }}=1.4 \times 10^{54} \mathrm{erg}$ ) and it occured relatively nearby (at redshift $z=0.34$ ). Therefore, it was extremely bright and in particular, it was detected by Fermi-LAT $(100 \mathrm{MeV}-100 \mathrm{GeV})$ for nearly a day, including a $95 \mathrm{GeV}$ photon several minutes after the burst and a $32 \mathrm{GeV}$ photon after 9 hours (Ackermann et al. 2014). Altogether, this GRB has a large number of high-energy photons that clearly violate (Ackermann et al. 2014) the maximum synchrotron photon energy limit ${ }^{13}$

$$
E_{\text {syn,max }} \sim \frac{\Gamma}{(1+z)} \frac{m_{e} c^{2}}{\alpha}=3.5\left(\frac{2}{1+z}\right) \frac{\Gamma}{100} \mathrm{GeV},
$$

which is obtained by equating the electron acceleration time to its synchrotron cooling time, assuming that it is accelerated and radiates its synchrotron emission in same

\footnotetext{
13 The exact numerical coefficient depends on the exact assumptions, and in particular on whether the acceleration time is assumed to be a fraction of or a complete Larmor gyration time, which is in any case a very fast acceleration, and arguably even unrealistically so. Here $\alpha \approx 1 / 137$ is the fine structure constant.
} 


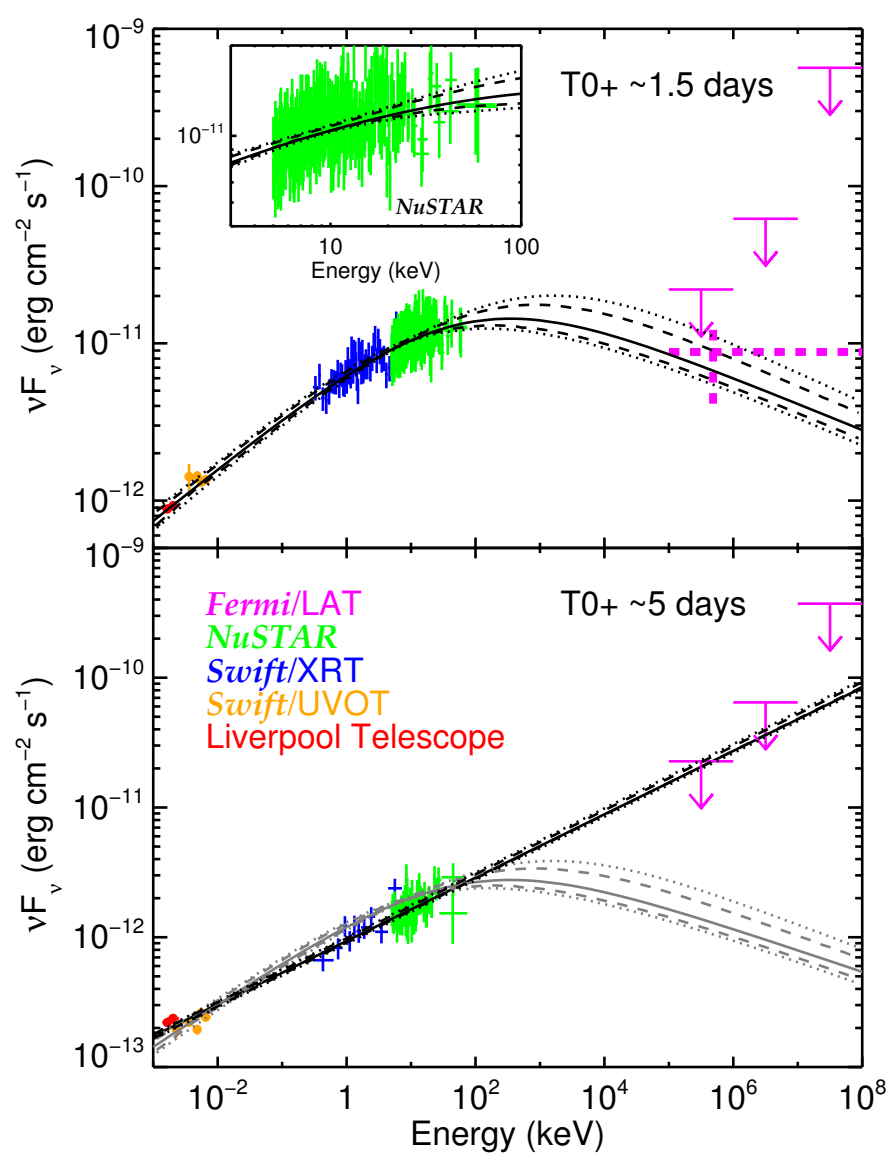

Fig. 12 The optical to GeV spectrum of GRB 130427A fit with the afterglow synchrotron model of Granot and Sari (2002). Broadband SEDs are shown during the first (top-panel) and the second (bottom-panel) NuSTAR epochs. The Fermi/LAT upper-limits are shown as arrows and the extrapolation of the LAT flux light curve is shown as a dashed magenta cross (only during the first epoch). The second epoch (bottompanel) is fit with a power law (black lines); the fit to the first epoch is scaled down and superposed on the second epoch data for comparison (in gray). (This figure is taken from Kouveliotou et al. 2013)

same magnetic field strength. This has been argued in order to rule out an afterglow synchrotron origin of the late-time high-energy LAT photons, and in particular motivated suggestions for an origin in a distinct SSC spectral component (e.g. Ackermann et al. 2014; Tam et al.2013; Liu et al. 2013; Fan et al.2013b).

However, Kouveliotou et al. (2013) have shown that the optical to GeV spectrum is consistent with a single spectral component that very nicely matches the expectation for synchrotron afterglow emission (Granot and Sari 2002, see Fig. 10). Figure 12 shows their spectral fit, and demonstrates that there is hardly any room for a distinct (SSC) spectral component to dominate the observed flux at the highest LAT energies (above several $\mathrm{GeV}$ or so, as is needed to avoid violating $E_{\mathrm{syn}, \max }$ ). This conclusion 
is strengthened by strict upper limits on the $>100 \mathrm{GeV}$ flux measured by VERITAS at three different epochs near the first NuSTAR observation (Aliu et al.|2014).

Therefore, this comprises very compelling evidence for a genuine violation of the $E_{\text {syn,max }}$ in this case, which is much harder to circumvent compared with previous Fermi/LAT GRBs (Abdo et al. 2009, Piran and Nakar 2010; Atwood et al. 2013). Thus, one should start to seriously consider how this limit can indeed be violated. A possible solution may lie in relaxing the assumption of a uniform magnetic field and instead allowing for a lower magnetic field acceleration region and a higher magnetic field synchrotron radiation region (e.g., Lyutikov 2010; Kumar et al. 2012). Such a situation might arise for diffusive shock acceleration (Fermi Type I) if the tangled shock-amplified magnetic field decays on a short length-scale behind the shock front (where most of the high-energy radiation is emitted), while the highest energy electrons are accelerated in the lower magnetic field further downstream (Kumar et al. 2012). In fact, such a scenario has recently been suggested (Lemoine 2013; Lemoine et al. 2013), and also shown to significantly alleviate the previously very high $\gamma$-ray radiative efficiencies inferred for most Swift GRBs (Beniamini et al.|2015).

\section{Conclusions}

In this review we have demonstrated that magnetic fields clearly play a vital role in GRBs, practically in every important aspect of this phenomenon. Here we briefly summarize some of our main conclusions, and stress both what was learned so far, as well as what still needs to be carefully studied. Following the main text, the discussion proceeds more or less in order of increasing distance from the central source.

Magnetic fields most likely play a crutial role in the launching of GRB jets. Moreover, hydromagnetic jet launching implies dynamically strong magnetic fields near the central source, which can naturally help avoid an excessive baryonic loading into the jet. Such a low baryon loading is essential in order for the jet to be able to reach sufficiently large Lorentz factors $(\Gamma \gtrsim 100-500)$ that are inferred from prompt GRB observations. The jet launching definitely requires many further detailed studies, also (semi-) analytic, but mainly numerical studies. The latter are, however, involved as they require general-relativistic magneto-hydrodynamics (GRMHD) codes, coupled with neutrino, plasma and radiation physics. Therefore, is it likely to take many years before such studies will provide definitive answers.

Millisecond-magnetar models for the GRB central engine, discussed in $\$ 2$, face serious challenges that still must be overcome. Models where the GRB arises from the delayed collapse of a supramassive millisecond magnetar (such as the "time reversal model"; Rezzolla and Kumar 2015; Ciolfi and Siegel 2015) face serious difficulty as this requires the formation of a disk during this collapse, which was recently argued to not be possible (Margalit et al. 2015). Producing both the prompt GRB emission and the X-ray plateau observed by Swift in long GRBs is challenging for millisecondmagnetar models. Models invoking millisecond magnetars in short GRBs also face many problems, such as how to produce the short GRB, or hide the huge amount of of rotational energy (a few $\times 10^{52} \mathrm{erg}$ ) that is injected into the afterglow shock, while short GRB afterglows are very dim. More detailed studies of the relevant physics, 
both analytic and numerical, are definitely needed in order to produce more robust and realistic predictions that could be tested in more detail against the relevant observations. A relatively simple example is that a highly-relativistic pulsar-like magnetar MHD wind is invoked to explain the X-ray plateaus observed by Swift. However, most studies consider only the forward shock emission and ignore the emission from the MHD wind itself (see, however, Dai 2004), which may involve magnetic reconnection and the resulting particle acceleration at the termination shock of the pulsar wind, similar to pulsar wind nebulae (see, e.g., Lyubarsky and Kirk 2001; Lyubarsky 2003; Sironi and Spitkovsky 2014). The birth process of a millisecond magnetar, either in the core-collapse of a massive star or in a binary neutron star merger, and how it evolves in these messy environments, is obviously very involved but there is definitely a lot of room for improvement and new studies on such systems.

The GRB outflow composition, and in particular its degree of magnetization is an important open question. On the one hand, in $\$ 3.1$ we have shown very compelling evidence based on the theoretical jet propagation time in the progenitor star of a long GRB and the observed GRB duration distribution, that the jet appears to have modest magnetization $(\sigma \lesssim 1)$ throughout most of the time it takes for it to bore its way out of the star. Moreover, in 4.5 we argued based on the prompt GRB observations (both $\gamma$ ray detections and upper limits in optical and soft X-ray) that the emission region has a low magnetization. On the other hand, hydromagnetic jet launching is much more promising than a pure thermal one, and can naturally help avoid excessive baryon loading, suggesting a high magnetization near the source $\left(\sigma_{0} \gg 1\right)$. A similar conclusion is also strongly suggested from the fact that quasi-thermal components in the prompt GRB spectrum are typically highly sub-dominant (see 84 ). Taking these two lines of evidence together, it appears that the magnetization significantly decreases as the jet propagates from the source out to large distances. This can occur through two main channels (or some combination of the two): (i) very efficient conversion of magnetic to kinetic energy that leaves a low magnetization in the emission region and allows for efficient internal shocks, through first quasi-steady and then impulsive magnetic acceleration (see Fig. 6, Granot et al. 2011; Granot 2012b), or (ii) strong magnetic reconnection that converts magnetic energy into thermal energy and accelerates particles, and yet somehow leaves a sufficiently low magnetization where these particles radiate most of their energy.

Magnetic fields can also play a very important role in the energy dissipation that leads to the GRB emission. On the one hand, sufficiently low magnetization $(\sigma<1$ or even $\sigma \lesssim 0.1$ ) is needed for efficient energy dissipation in internal shocks. On the other hand, while a large magnetization $(\sigma>1)$ effectively suppresses internal shocks, or the reverse shock, it can lead to significant dissipation through magnetic reconnection under appropriate conditions (e.g. if the source ejects outflow with a magnetic field that occasionally changes its polarity, or through certain instabilities).

Another vital role of magnetic fields in GRBs is in particle acceleration. Within the outflow the magnetization can be high, allowing efficient magnetic reconnection, which can directly convert a good fraction of the dissipated magnetic energy into the random motions of the particles that it accelerates in this process. The exact magnetic field strength and geometry throughout the emission region also greatly affect the radiation of the accelerated particles, and therefore their observable signatures. In 
particular, magnetic fields are vital to the synchrotron emission, which dominates in the afterglow and also likely plays a key role in the prompt GRB emission.

Both optically thin internal shocks as well as the external forward and reverse shocks are collisionless, and mediated by electromagnetic fields (through collective plasma interactions). Moreover, while the outflow itself typically has a large enough magnetic field for efficient synchrotron radiation, the external medium has a very low magnetization that must be significantly amplified at the afterglow shock front in order to produce the observed afterglow emission. Therefore, magnetic field amplification in relativistic collisionless shocks and its possible decay behind the shock are vital for understanding the afterglow physics and interpreting afterglow observations. Moreover they can strongly affect the particle acceleration in the afterglow shock, and the resulting afterglow emission. Such physics might hold the key to unravel a puzzle (see $\$ 5.3$ ) arising from observations of the very bright and relatively nearby long GRB 130427A, which show an apparent violation of the maximum synchrotron photon energy limit, $E_{\mathrm{syn}, \max }$.

The different roles of magnetic fields in GRBs are numerous and diverse. Many of them are only starting to be understood, while new roles are still being occasionally discovered. Since magnetic fields appear in almost all aspects of GRB physics, future studies of their properties and effects are likely to greatly improve our understanding of GRBs, and lead to fundamental progress.

Acknowledgements The authors acknowledge support from the ISF grant 719/14 (JG), as well as from the I-CORE Program - ISF grant 1829/12, the ISA grant 3-10417, and an ISF-CNSF grant (TP).

\section{References}

A.A. Abdo, et al., Fermi Observations of GRB 090902B: A Distinct Spectral Component in the Prompt and Delayed Emission. Astrophys. J. Lett. 706, 138-144 (2009). doi:10.1088/0004-637X/706/1/L138

M. Ackermann, et al., Fermi Observations of GRB 090510: A Short-Hard Gamma-ray Burst with an Additional, Hard Power-law Component from $10 \mathrm{keV}$ TO GeV Energies. Astrophys. J. 716, 11781190 (2010). doi:10.1088/0004-637X/716/2/1178

M. Ackermann, et al., The First Fermi-LAT Gamma-Ray Burst Catalog. Astrophys. J. Supplements 209, 11 (2013). doi:10.1088/0067-0049/209/1/11

M. Ackermann, et al., Fermi-LAT Observations of the Gamma-Ray Burst GRB 130427A. Science 343, 42-47 (2014). doi:10.1126/science. 1242353

C. Akerlof, et al., Observation of contemporaneous optical radiation from a $\gamma$-ray burst. Nature 398, 400402 (1999). doi:10.1038/18837

E. Aliu, et al., Constraints on Very High Energy Emission from GRB 130427A. Astrophys. J. Lett. 795, 3 (2014). doi:10.1088/2041-8205/795/1/L3

K. Asano, P. Mészáros, Spectral-Temporal Simulations of Internal Dissipation Models of Gamma-Ray Bursts. Astrophys. J. 739, 103 (2011). doi:10.1088/0004-637X/739/2/103

K. Asano, P. Mészáros, Delayed Onset of High-energy Emissions in Leptonic and Hadronic Models of Gamma-Ray Bursts. Astrophys. J. 757, 115 (2012). doi:10.1088/0004-637X/757/2/115

W.B. Atwood, et al., New Fermi-LAT Event Reconstruction Reveals More High-energy Gamma Rays from Gamma-Ray Bursts. Astrophys. J. 774, 76 (2013). doi:10.1088/0004-637X/774/1/76 
M. Axelsson, et al., GRB110721A: An Extreme Peak Energy and Signatures of the Photosphere. Astrophys. J. Lett. 757, 31 (2012). doi:10.1088/2041-8205/757/2/L31

D. Band, et al., BATSE observations of gamma-ray burst spectra. I - Spectral diversity. Astrophys. J. 413, 281-292 (1993). doi:10.1086/172995

M.G. Baring, A.K. Harding, The Escape of High-Energy Photons from Gamma-Ray Bursts. Astrophys. J. 491, 663-686 (1997)

R. Barniol Duran, Ž. Bošnjak, P. Kumar, Inverse-Compton cooling in Klein-Nishina regime and gammaray burst prompt spectrum. MNRAS 424, 3192-3200 (2012). doi:10.1111/j.1365-2966.2012.21533.x

C. Barraud, F. Daigne, R. Mochkovitch, J.L. Atteia, On the nature of X-ray flashes. Astron. Astrophys. 440, 809-817 (2005). doi:10.1051/0004-6361:20041572

M.C. Begelman, Instability of Toroidal Magnetic Field in Jets and Plerions. Astrophys. J. 493, 291 (1998). doi: $10.1086 / 305119$

A.M. Beloborodov, Collisional mechanism for gamma-ray burst emission. MNRAS 407, 1033-1047 (2010). doi:10.1111/j.1365-2966.2010.16770.x

A.M. Beloborodov, Radiative Transfer in Ultrarelativistic Outflows. Astrophys. J. 737, 68 (2011). doi: $10.1088 / 0004-637$ X/737/2/68

A.M. Beloborodov, Regulation of the Spectral Peak in Gamma-Ray Bursts. Astrophys. J. 764, 157 (2013). doi:10.1088/0004-637X/764/2/157

A.M. Beloborodov, R. Hascoët, I. Vurm, On the Origin of GeV Emission in Gamma-Ray Bursts. Astrophys. J. 788, 36 (2014). doi:10.1088/0004-637X/788/1/36

A.M. Beloborodov, B.E. Stern, R. Svensson, Power Density Spectra of Gamma-Ray Bursts. Astrophys. J. 535, 158-166 (2000). doi:10.1086/308836

P. Beniamini, T. Piran, Constraints on the Synchrotron Emission Mechanism in Gamma-Ray Bursts. Astrophys. J. 769, 69 (2013). doi:10.1088/0004-637X/769/1/69

P. Beniamini, T. Piran, The emission mechanism in magnetically dominated gamma-ray burst outflows MNRAS 445, 3892-3907 (2014). doi:10.1093/mnras/stu2032

P. Beniamini, L. Nava, R. Barniol Duran, T. Piran, Energies of GRB blast waves and prompt efficiencies as implied by self-consistent modeling of X-ray and LAT afterglows. ArXiv e-prints (2015)

E. Berger, A.M. Soderberg, D.A. Frail, S.R. Kulkarni, A Radio Flare from GRB 020405: Evidence for a Uniform Medium around a Massive Stellar Progenitor. Astrophys. J. Lett. 587, 5-8 (2003). doi: $10.1086 / 375158$

R.D. Blandford, To the Lighthouse, in Lighthouses of the Universe: The Most Luminous Celestial Objects and Their Use for Cosmology, ed. by M. Gilfanov, R. Sunyeav, E. Churazov, 2002, p. 381. doi:10.1007/10856495_59

R.D. Blandford, C.F. McKee, Fluid dynamics of relativistic blast waves. Physics of Fluids 19, 1130-1138 (1976). doi:10.1063/1.861619

J.S. Bloom, D.A. Frail, S.R. Kulkarni, Gamma-Ray Burst Energetics and the Gamma-Ray Burst Hubble Diagram: Promises and Limitations. Astrophys. J. 594, 674-683 (2003). doi:10.1086/377125

Ž. Bošnjak, F. Daigne, Spectral evolution in gamma-ray bursts: Predictions of the internal shock model and comparison to observations. Astron. Astrophys. 568, 45 (2014). doi:10.1051/0004-6361/201322341

Ž. Bošnjak, F. Daigne, G. Dubus, Prompt high-energy emission from gamma-ray bursts in the internal shock model. Astron. Astrophys. 498, 677-703 (2009). doi:10.1051/0004-6361/200811375

O. Bromberg, A. Levinson, Hydrodynamic Collimation of Relativistic Outflows: Semianalytic Solutions and Application to Gamma-Ray Bursts. Astrophys. J. 671, 678-688 (2007). doi:10.1086/522668

O. Bromberg, J. Granot, T. Piran, On the composition of GRBs' Collapsar jets. MNRAS 450, 1077-1084 (2015). doi:10.1093/mnras/stv226

O. Bromberg, E. Nakar, T. Piran, R. Sari, The Propagation of Relativistic Jets in External Media. Astrophys. J. 740, 100 (2011). doi:10.1088/0004-637X/740/2/100

O. Bromberg, E. Nakar, T. Piran, R. Sari, An Observational Imprint of the Collapsar Model of Long Gamma-Ray Bursts. Astrophys. J. 749, 110 (2012). doi:10.1088/0004-637X/749/2/110

O. Bromberg, E. Nakar, T. Piran, R. Sari, Short versus Long and Collapsars versus Non-collapsars: A Quantitative Classification of Gamma-Ray Bursts. Astrophys. J. 764, 179 (2013). doi:10.1088/0004 637X/764/2/179

O. Bromberg, J. Granot, Y. Lyubarsky, T. Piran, The dynamics of a highly magnetized jet propagating inside a star. MNRAS 443, 1532-1548 (2014). doi:10.1093/mnras/stu995

N. Bucciantini, E. Quataert, J. Arons, B.D. Metzger, T.A. Thompson, Magnetar-driven bubbles and the origin of collimated outflows in gamma-ray bursts. MNRAS 380, 1541-1553 (2007) doi:10.1111/j.1365-2966.2007.12164.x 
N. Bucciantini, E. Quataert, J. Arons, B.D. Metzger, T.A. Thompson, Relativistic jets and long-duration gamma-ray bursts from the birth of magnetars. MNRAS 383, 25-29 (2008). doi:10.1111/j.17453933.2007.00403.x

N. Bucciantini, E. Quataert, B.D. Metzger, T.A. Thompson, J. Arons, L. Del Zanna, Magnetized relativistic jets and long-duration GRBs from magnetar spin-down during core-collapse supernovae. MNRAS 396, 2038-2050 (2009). doi:10.1111/j.1365-2966.2009.14940.x

N. Bucciantini, B.D. Metzger, T.A. Thompson, E. Quataert, Short gamma-ray bursts with extended emission from magnetar birth: jet formation and collimation. MNRAS 419, 1537-1545 (2012). doi:10.1111/j.1365-2966.2011.19810.x

J.M. Burgess, et al., Constraints on the Synchrotron Shock Model for the Fermi GRB 090820A Observed by Gamma-Ray Burst Monitor. Astrophys. J. 741, 24 (2011). doi:10.1088/0004-637X/741/1/24

J.M. Burgess, et al., Time-resolved Analysis of Fermi Gamma-Ray Bursts with Fast- and Slow-cooled Synchrotron Photon Models. Astrophys. J. 784, 17 (2014). doi:10.1088/0004-637X/784/1/17

A.M. Bykov, P. Meszaros, Electron Acceleration and Efficiency in Nonthermal Gamma-Ray Sources. Astrophys. J. Lett. 461, 37 (1996). doi:10.1086/309999

A. Bykov, N. Gehrels, H. Krawczynski, M. Lemoine, G. Pelletier, M. Pohl, Particle Acceleration in Relativistic Outflows. Space Science Reviews 173, 309-339 (2012). doi:10.1007/s11214-012-9896-y

S. Campana, et al., The association of GRB 060218 with a supernova and the evolution of the shock wave. Nature 442, 1008-1010 (2006). doi:10.1038/nature04892

S.B. Cenko, et al., The Collimation and Energetics of the Brightest Swift Gamma-ray Bursts. Astrophys. J. 711, 641-654 (2010). doi:10.1088/0004-637X/711/2/641

R. Ciolfi, D.M. Siegel, Short Gamma-Ray Bursts in the "Time-reversal” Scenario. Astrophys. J. Lett. 798, 36 (2015). doi:10.1088/2041-8205/798/2/L36

W. Coburn, S.E. Boggs, Polarization of the prompt $\gamma$-ray emission from the $\gamma$-ray burst of 6 December 2002. Nature 423, 415-417 (2003). doi:10.1038/nature01612

E. Cohen, J.I. Katz, T. Piran, R. Sari, R.D. Preece, D.L. Band, Possible Evidence for Relativistic Shocks in Gamma-Ray Bursts. Astrophys. J. 488, 330-337 (1997)

J. Contopoulos, A Simple Type of Magnetically Driven Jets: an Astrophysical Plasma Gun. Astrophys. J. 450, 616 (1995). doi:10.1086/176170

S. Covino, G. Ghisellini, D. Lazzati, D. Malesani, Polarization of Gamma-Ray Burst Optical and NearInfrared Afterglows, in Gamma-Ray Bursts in the Afterglow Era, ed. by M. Feroci, F. Frontera, N. Masetti, L. Piro Astronomical Society of the Pacific Conference Series, vol. 312, 2004, p. 169

Z.G. Dai, Relativistic Wind Bubbles and Afterglow Signatures. Astrophys. J. 606, 1000-1005 (2004). doi:10.1086/383019

F. Daigne, R. Mochkovitch, Gamma-ray bursts from internal shocks in a relativistic wind: temporal and spectral properties. MNRAS 296, 275-286 (1998). doi:10.1046/j.1365-8711.1998.01305.x

F. Daigne, R. Mochkovitch, Gamma-ray bursts from internal shocks in a relativistic wind: a hydrodynamical study. Astron. Astrophys. 358, 1157-1166 (2000)

F. Daigne, R. Mochkovitch, The expected thermal precursors of gamma-ray bursts in the internal shock model. MNRAS 336, 1271-1280 (2002). doi:10.1046/j.1365-8711.2002.05875.x

F. Daigne, R. Mochkovitch, The physics of pulses in gamma-ray bursts: emission processes, temporal profiles and time-lags. MNRAS 342, 587-592 (2003). doi:10.1046/j.1365-8711.2003.06575.x

F. Daigne, Ž. Bošnjak, G. Dubus, Reconciling observed gamma-ray burst prompt spectra with synchrotron radiation? Astron. Astrophys. 526, 110 (2011). doi:10.1051/0004-6361/201015457

S. Dall'Osso, J. Granot, T. Piran, Magnetic field decay in neutron stars: from soft gamma repeaters to 'weak-field magnetars'. MNRAS 422, 2878-2903 (2012). doi:10.1111/j.1365-2966.2012.20612.x

S. Dall'Osso, S.N. Shore, L. Stella, Early evolution of newly born magnetars with a strong toroidal field. MNRAS 398, 1869-1885 (2009). doi:10.1111/j.1365-2966.2008.14054.x

S. Dall'Osso, G. Stratta, D. Guetta, S. Covino, G. De Cesare, L. Stella, Gamma-ray bursts afterglows with energy injection from a spinning down neutron star. Astron. Astrophys. 526, 121 (2011) doi:10.1051/0004-6361/201014168

W. Deng, B. Zhang, Low Energy Spectral Index and $\mathrm{E}_{p}$ Evolution of Quasi-thermal Photosphere Emission of Gamma-Ray Bursts. Astrophys. J. 785, 112 (2014). doi:10.1088/0004-637X/785/2/112

E.V. Derishev, Synchrotron emission in the fast cooling regime: which spectra can be explained? Ap \& SS 309, 157-161 (2007). doi:10.1007/s10509-007-9421-z

E.V. Derishev, V.V. Kocharovsky, V.V. Kocharovsky, Physical parameters and emission mechanism in gamma-ray bursts. Astron. Astrophys. 372, 1071-1077 (2001). doi:10.1051/0004-6361:20010586

G. Drenkhahn, H.C. Spruit, Efficient acceleration and radiation in Poynting flux powered GRB outflows. 
Astron. Astrophys. 391, 1141-1153 (2002). doi:10.1051/0004-6361:20020839

R.C. Duncan, C. Thompson, Formation of very strongly magnetized neutron stars - Implications for gamma-ray bursts. Astrophys. J. Lett. 392, 9-13 (1992). doi:10.1086/186413

D. Eichler, Magnetic Confinement of Jets. Astrophys. J. 419, 111 (1993). doi:10.1086/173464

D. Eichler, J. Granot, The Case for Anisotropic Afterglow Efficiency within Gamma-Ray Burst Jets. Astrophys. J. Lett. 641, 5-8 (2006). doi:10.1086/503667

D. Eichler, E. Waxman, The Efficiency of Electron Acceleration in Collisionless Shocks and Gamma-Ray Burst Energetics. Astrophys. J. 627, 861-867 (2005). doi:10.1086/430596

D. Eichler, M. Livio, T. Piran, D.N. Schramm, Nucleosynthesis, neutrino bursts and gamma-rays from coalescing neutron stars. Nature 340, 126-128 (1989). doi:10.1038/340126a0

Y.-Z. Fan, The spectrum of $\gamma$-ray burst: a clue. MNRAS 403, 483-490 (2010). doi:10.1111/j.13652966.2009.16134.x

Y.-Z. Fan, et al., A Supramassive Magnetar Central Engine for GRB 130603B. Astrophys. J. Lett. 779, 25 (2013a). doi:10.1088/2041-8205/779/2/L25

Y.-Z. Fan, et al., High-energy Emission of GRB 130427A: Evidence for Inverse Compton Radiation. Astrophys. J. 776, 95 (2013b). doi:10.1088/0004-637X/776/2/95

Y.-Z. Fan, D. Xu, The X-ray afterglow flat segment in short GRB 051221A: Energy injection from a millisecond magnetar? MNRAS 372, 19-22 (2006). doi:10.1111/j.1745-3933.2006.00217.x

D.A. Frail, et al., The Enigmatic Radio Afterglow of GRB 991216. Astrophys. J. Lett. 538, 129-132 (2000). doi:10.1086/312807

H. Gao, B.-B. Zhang, B. Zhang, Stepwise Filter Correlation Method and Evidence of Superposed Variability Components in Gamma-Ray Burst Prompt Emission Light Curves. Astrophys. J. 748, 134 (2012). doi:10.1088/0004-637X/748/2/134

B. Gendre, et al., Testing gamma-ray burst models with the afterglow of GRB 090102. MNRAS 405, 2372-2380 (2010). doi:10.1111/j.1365-2966.2010.16601.x

F. Genet, J. Granot, Realistic analytic model for the prompt and high-latitude emission in GRBs. MNRAS 399, 1328-1346 (2009). doi:10.1111/j.1365-2966.2009.15355.x

F. Genet, F. Daigne, R. Mochkovitch, Can the early X-ray afterglow of gamma-ray bursts be explained by a contribution from the reverse shock? MNRAS 381, 732-740 (2007). doi:10.1111/j.13652966.2007.12243.x

G. Ghisellini, Jetted Active Galactic Nuclei. International Journal of Modern Physics Conference Series 8, 1-12 (2012). doi:10.1142/S2010194512004345

G. Ghisellini, A. Celotti, Quasi-thermal Comptonization and Gamma-Ray Bursts. Astrophys. J. Lett. 511, 93-96 (1999). doi:10.1086/311845

G. Ghisellini, D. Lazzati, Polarization light curves and position angle variation of beamed gamma-ray bursts. MNRAS 309, 7-11 (1999). doi:10.1046/j.1365-8711.1999.03025.x

G. Ghisellini, A. Celotti, D. Lazzati, Constraints on the emission mechanisms of gamma-ray bursts. MNRAS 313, 1-5 (2000). doi:10.1046/j.1365-8711.2000.03354.x

D. Giannios, Prompt GRB emission from gradual energy dissipation. Astron. Astrophys. 480, 305-312 (2008). doi:10.1051/0004-6361:20079085

D. Giannios, H.C. Spruit, The role of kink instability in Poynting-flux dominated jets. Astron. Astrophys. 450, 887-898 (2006). doi:10.1051/0004-6361:20054107

D. Giannios, H.C. Spruit, Spectral and timing properties of a dissipative $\gamma$-ray burst photosphere. Astron. Astrophys. 469, 1-9 (2007). doi:10.1051/0004-6361:20066739

P. Goldreich, W.H. Julian, Stellar Winds. Astrophys. J. 160, 971 (1970). doi:10.1086/150486

B.P. Gompertz, P.T. O'Brien, G.A. Wynn, Magnetar powered GRBs: explaining the extended emission and X-ray plateau of short GRB light curves. MNRAS 438, 240-250 (2014). doi:10.1093/mnras/stt2165

J. Goodman, Are gamma-ray bursts optically thick? Astrophys. J. Lett. 308, 47-50 (1986). doi:10.1086/184741

D. Götz, et al., GRB 140206A: the most distant polarized gamma-ray burst. MNRAS 444, 2776-2782 (2014). doi:10.1093/mnras/stu1634

D. Götz, P. Laurent, F. Lebrun, F. Daigne, Ž. Bošnjak, Variable Polarization Measured in the Prompt Emission of GRB 041219A Using IBIS on Board INTEGRAL. Astrophys. J. Lett. 695, 208-212 (2009). doi:10.1088/0004-637X/695/2/L208

J. Granot, The Most Probable Cause for the High Gamma-Ray Polarization in GRB 021206. Astrophys. J. Lett. 596, 17-21 (2003). doi:10.1086/379110

J. Granot, Interaction of a highly magnetized impulsive relativistic flow with an external medium. MNRAS 421, 2442-2466 (2012a). doi:10.1111/j.1365-2966.2012.20473.x 
J. Granot, The effects of sub-shells in highly magnetized relativistic flows. MNRAS 421, 2467-2477 (2012b). doi:10.1111/j.1365-2966.2012.20474.x

J. Granot, A. Königl, Linear Polarization in Gamma-Ray Bursts: The Case for an Ordered Magnetic Field. Astrophys. J. Lett. 594, 83-87 (2003). doi:10.1086/378733

J. Granot, P. Kumar, Distribution of gamma-ray burst ejecta energy with Lorentz factor. MNRAS 366, 13-16 (2006). doi:10.1111/j.1745-3933.2005.00121.x

J. Granot, R. Sari, The Shape of Spectral Breaks in Gamma-Ray Burst Afterglows. Astrophys. J. 568 , 820-829 (2002). doi:10.1086/338966

J. Granot, G.B. Taylor, Radio Flares and the Magnetic Field Structure in Gamma-Ray Burst Outflows. Astrophys. J. 625, 263-270 (2005). doi:10.1086/429536

J. Granot, J. Cohen-Tanugi, E. do Couto e Silva, Opacity Buildup in Impulsive Relativistic Sources. Astrophys. J. 677, 92-126 (2008). doi:10.1086/526414

J. Granot, S.S. Komissarov, A. Spitkovsky, Impulsive acceleration of strongly magnetized relativistic flows. MNRAS 411, 1323-1353 (2011). doi:10.1111/j.1365-2966.2010.17770.x

J. Granot, A. Königl, T. Piran, Implications of the early X-ray afterglow light curves of Swift gamma-ray bursts. MNRAS 370, 1946-1960 (2006). doi:10.1111/j.1365-2966.2006.10621.x

D. Gruber, et al., The Fermi GBM Gamma-Ray Burst Spectral Catalog: Four Years of Data. Astrophys. J. Supplements 211, 12 (2014). doi:10.1088/0067-0049/211/1/12

A. Gruzinov, E. Waxman, Gamma-Ray Burst Afterglow: Polarization and Analytic Light Curves. Astrophys. J. 511, 852-861 (1999). doi:10.1086/306720

D. Guetta, J. Granot, High-Energy Emission from the Prompt Gamma-Ray Burst. Astrophys. J. 585, 885889 (2003). doi:10.1086/346221

C. Guidorzi, et al., Average power density spectrum of Swift long gamma-ray bursts in the observer and in the source-rest frames. MNRAS 422, 1785-1803 (2012). doi:10.1111/j.1365-2966.2012.20758.x

S. Guiriec, et al., Time-resolved Spectroscopy of the Three Brightest and Hardest Short Gamma-ray Bursts Observed with the Fermi Gamma-ray Burst Monitor. Astrophys. J. 725, 225-241 (2010). doi:10.1088/0004-637X/725/1/225

S. Guiriec, et al., Detection of a Thermal Spectral Component in the Prompt Emission of GRB 100724B. Astrophys. J. Lett. 727, 33 (2011). doi:10.1088/2041-8205/727/2/L33

S. Guiriec, et al., Evidence for a Photospheric Component in the Prompt Emission of the Short GRB 120323A and Its Effects on the GRB Hardness-Luminosity Relation. Astrophys. J. 770, 32 (2013). doi:10.1088/0004-637X/770/1/32

S. Guiriec, et al., Towards a Better Understanding of the GRB Phenomenon: a New Model for GRB Prompt Emission and its effects on the New Non-Thermal $L_{\mathrm{i}}^{\mathrm{NT}}-E_{\text {peak,i }}^{\text {rest,NT }}$ relation. ArXiv e-prints (2015)

F.A. Harrison, et al., Broadband Observations of the Afterglow of GRB 000926: Observing the Effect of Inverse Compton Scattering. Astrophys. J. 559, 123-130 (2001). doi:10.1086/322368

R. Hascoët, F. Daigne, R. Mochkovitch, Accounting for the XRT early steep decay in models of the prompt gamma-ray burst emission. Astron. Astrophys. 542, 29 (2012). doi:10.1051/0004-6361/201219339

R. Hascoët, F. Daigne, R. Mochkovitch, Prompt thermal emission in gamma-ray bursts. Astron. Astrophys 551, 124 (2013). doi:10.1051/0004-6361/201220023

R. Hascoët, F. Daigne, R. Mochkovitch, The prompt-early afterglow connection in gamma-ray bursts: implications for the early afterglow physics. MNRAS 442, 20-27 (2014). doi:10.1093/mnras/stu750

R. Hascoët, F. Daigne, R. Mochkovitch, V. Vennin, Do Fermi Large Area Telescope observations imply very large Lorentz factors in gamma-ray burst outflows? MNRAS 421, 525-545 (2012). doi:10.1111/j.1365-2966.2011.20332.x

R. Hascoet, A.M. Beloborodov, F. Daigne, R. Mochkovitch, X-ray flares from dense shells formed in gamma-ray burst explosions. ArXiv e-prints (2015)

S. Heinz, M.C. Begelman, Jet Acceleration by Tangled Magnetic Fields. Astrophys. J. 535, 104-117 (2000). doi: $10.1086 / 308820$

D. Kagan, L. Sironi, B. Cerutti, D. Giannios, Relativistic Magnetic Reconnection in Pair Plasmas and Its Astrophysical Applications. Space Science Reviews (2015). doi:10.1007/s11214-014-0132-9

Y. Kaneko, R.D. Preece, M.S. Briggs, W.S. Paciesas, C.A. Meegan, D.L. Band, The Complete Spectral Catalog of Bright BATSE Gamma-Ray Bursts. Astrophys. J. Supplements 166, 298-340 (2006). doi:10.1086/505911

N. Kawanaka, T. Piran, J.H. Krolik, Jet Luminosity from Neutrino-dominated Accretion Flows in GammaRay Bursts. Astrophys. J. 766, 31 (2013). doi:10.1088/0004-637X/766/1/31

U. Keshet, B. Katz, A. Spitkovsky, E. Waxman, Magnetic Field Evolution in Relativistic Unmagnetized 
Collisionless Shocks. Astrophys. J. Lett. 693, 127-130 (2009). doi:10.1088/0004-637X/693/2/L127

W. Kluźniak, M. Ruderman, The Central Engine of Gamma-Ray Bursters. Astrophys. J. Lett. 505, 113 117 (1998). doi:10.1086/311622

S. Kobayashi, T. Piran, R. Sari, Can Internal Shocks Produce the Variability in Gamma-Ray Bursts? Astrophys. J. 490, 92 (1997). doi:10.1086/512791

S.S. Komissarov, Magnetic acceleration of relativistic jets. Mem. Soc. Astron. Italiana 82, 95 (2011)

S.S. Komissarov, Shock dissipation in magnetically dominated impulsive flows. MNRAS 422, 326-346 (2012). doi:10.1111/j.1365-2966.2012.20609.x

S.S. Komissarov, N. Vlahakis, A. Königl, Rarefaction acceleration of ultrarelativistic magnetized jets in gamma-ray burst sources. MNRAS 407, 17-28 (2010). doi:10.1111/j.1365-2966.2010.16779.x

S.S. Komissarov, N. Vlahakis, A. Königl, M.V. Barkov, Magnetic acceleration of ultrarelativistic jets in gamma-ray burst sources. MNRAS 394, 1182-1212 (2009). doi:10.1111/j.1365-2966.2009.14410.x

C. Kouveliotou, et al., NuSTAR Observations of GRB 130427A Establish a Single Component Synchrotron Afterglow Origin for the Late Optical to Multi-GeV Emission. Astrophys. J. Lett. 779, 1 (2013). doi:10.1088/2041-8205/779/1/L1

C. Kouveliotou, C.A. Meegan, G.J. Fishman, N.P. Bhat, M.S. Briggs, T.M. Koshut, W.S. Paciesas, G.N. Pendleton, Identification of two classes of gamma-ray bursts. Astrophys. J. Lett. 413, 101-104 (1993). doi:10.1086/186969

S.R. Kulkarni, et al., Discovery of a Radio Flare from GRB 990123. Astrophys. J. Lett. 522, 97-100 (1999). doi:10.1086/312227

P. Kumar, E. McMahon, A general scheme for modelling $\gamma$-ray burst prompt emission. MNRAS 384, 33-63 (2008). doi:10.1111/j.1365-2966.2007.12621.x

P. Kumar, A. Panaitescu, Afterglow Emission from Naked Gamma-Ray Bursts. Astrophys. J. Lett. 541, 51-54 (2000). doi:10.1086/312905

P. Kumar, B. Zhang, The physics of gamma-ray bursts \& relativistic jets. Physical Rept. 561, 1-109 (2015). doi:10.1016/j.physrep.2014.09.008

P. Kumar, R.A. Hernández, Ž. Bošnjak, R. Barniol Duran, Maximum synchrotron frequency for shockaccelerated particles. MNRAS 427, 40-44 (2012). doi:10.1111/j.1745-3933.2012.01341.x

A. Lazar, E. Nakar, T. Piran, Gamma-Ray Burst Light Curves in the Relativistic Turbulence and Relativistic Subjet Models. Astrophys. J. Lett. 695, 10-14 (2009). doi:10.1088/0004-637X/695/1/L10

M. Lemoine, Synchrotron signature of a relativistic blast wave with decaying microturbulence. MNRAS 428, 845-866 (2013). doi:10.1093/mnras/sts081

M. Lemoine, Z. Li, X.-Y. Wang, On the magnetization of gamma-ray burst blast waves. MNRAS 435, 3009-3016 (2013). doi:10.1093/mnras/stt1494

A. Levinson, Interaction of a Magnetized Shell with an Ambient Medium: Limits on Impulsive Magnetic Acceleration. Astrophys. J. 720, 1490-1499 (2010). doi:10.1088/0004-637X/720/2/1490

A. Levinson, D. Eichler, Baryon Purity in Cosmological Gamma-Ray Bursts as a Manifestation of Event Horizons. Astrophys. J. 418, 386 (1993). doi:10.1086/173397

A. Levinson, N. Globus, Ultra-relativistic, Neutrino-driven Flows in Gamma-Ray Bursts: A Double Transonic Flow Solution in a Schwarzschild Spacetime. Astrophys. J. 770, 159 (2013). doi:10.1088/0004$637 \mathrm{X} / 770 / 2 / 159$

E.W. Liang, et al., Testing the Curvature Effect and Internal Origin of Gamma-Ray Burst Prompt Emissions and X-Ray Flares with Swift Data. Astrophys. J. 646, 351-357 (2006). doi:10.1086/504684

E. Liang, B. Zhang, F. Virgili, Z.G. Dai, Low-Luminosity Gamma-Ray Bursts as a Unique Population: Luminosity Function, Local Rate, and Beaming Factor. Astrophys. J. 662, 1111-1118 (2007). doi: $10.1086 / 517959$

C.C. Lindner, M. Milosavljević, S.M. Couch, P. Kumar, Collapsar Accretion and the Gamma-Ray Burst X-Ray Light Curve. Astrophys. J. 713, 800-815 (2010). doi:10.1088/0004-637X/713/2/800

Y. Lithwick, R. Sari, Lower Limits on Lorentz Factors in Gamma-Ray Bursts. Astrophys. J. 555, 540-545 (2001). doi:10.1086/321455

R.-Y. Liu, X.-Y. Wang, X.-F. Wu, Interpretation of the Unprecedentedly Long-lived High-energy Emission of GRB 130427A. Astrophys. J. Lett. 773, 20 (2013). doi:10.1088/2041-8205/773/2/L20

A. Loeb, R. Perna, Microlensing of Gamma-Ray Burst Afterglows. Astrophys. J. 495, 597-603 (1998). doi: $10.1086 / 305337$

R.-J. Lu, et al., A Comprehensive Analysis of Fermi Gamma-Ray Burst Data. II. E $p$ Evolution Patterns and Implications for the Observed Spectrum-Luminosity Relations. Astrophys. J. 756, 112 (2012). doi:10.1088/0004-637X/756/2/112

C. Lundman, A. Pe'er, F. Ryde, A theory of photospheric emission from relativistic, collimated outflows. 
MNRAS 428, 2430-2442 (2013). doi:10.1093/mnras/sts219

Y.E. Lyubarskii, Kink instability of relativistic force-free jets. MNRAS 308, 1006-1010 (1999). doi:10.1046/j.1365-8711.1999.02763.x

Y.E. Lyubarskij, Energy release in strongly magnetized relativistic winds. Soviet Astronomy Letters 18, $356(1992)$

Y. Lyubarsky, Asymptotic Structure of Poynting-Dominated Jets. Astrophys. J. 698, 1570-1589 (2009). doi:10.1088/0004-637X/698/2/1570

Y. Lyubarsky, J.G. Kirk, Reconnection in a Striped Pulsar Wind. Astrophys. J. 547, 437-448 (2001). doi: $10.1086 / 318354$

Y.E. Lyubarsky, The termination shock in a striped pulsar wind. MNRAS 345, 153-160 (2003). doi:10.1046/j.1365-8711.2003.06927.x

Y.E. Lyubarsky, A New Mechanism for Dissipation of Alternating Fields in Poynting-dominated Outflows. Astrophys. J. Lett. 725, 234-238 (2010a). doi:10.1088/2041-8205/725/2/L234

Y.E. Lyubarsky, Transformation of the Poynting flux into kinetic energy in relativistic jets. MNRAS 402, 353-361 (2010b). doi:10.1111/j.1365-2966.2009.15877.x

M. Lyutikov, The electromagnetic model of gamma-ray bursts. New Journal of Physics 8, 119 (2006). doi:10.1088/1367-2630/8/7/119

M. Lyutikov, A high-sigma model of pulsar wind nebulae. MNRAS 405, 1809-1815 (2010) doi:10.1111/j.1365-2966.2010.16553.x

M. Lyutikov, Dynamics of strongly magnetized ejecta in gamma-ray bursts. MNRAS 411, 422-426 (2011). doi:10.1111/j.1365-2966.2010.17696.x

M. Lyutikov, R. Blandford, Gamma Ray Bursts as Electromagnetic Outflows. ArXiv Astrophysics e-prints (2003)

M. Lyutikov, V.I. Pariev, R.D. Blandford, Polarization of Prompt Gamma-Ray Burst Emission: Evidence for Electromagnetically Dominated Outflow. Astrophys. J. 597, 998-1009 (2003). doi: $10.1086 / 378497$

A.I. MacFadyen, S.E. Woosley, Collapsars: Gamma-Ray Bursts and Explosions in "Failed Supernovae". Astrophys. J. 524, 262-289 (1999). doi:10.1086/307790

B. Margalit, B.D. Metzger, A.M. Beloborodov, Does the Collapse of a Supramassive Neutron Star Leave a Debris Disk? ArXiv e-prints (2015)

A. Maselli, et al., GRB 130427A: A Nearby Ordinary Monster. Science 343, 48-51 (2014). doi:10.1126/science. 1242279

C.D. Matzner, Supernova hosts for gamma-ray burst jets: dynamical constraints. MNRAS 345, 575-589 (2003). doi:10.1046/j.1365-8711.2003.06969.x

S. McGlynn, et al., Polarisation studies of the prompt gamma-ray emission from GRB 041219a using the spectrometer aboard INTEGRAL. Astron. Astrophys. 466, 895-904 (2007). doi:10.1051/00046361:20066179

J.C. McKinney, D.A. Uzdensky, A reconnection switch to trigger gamma-ray burst jet dissipation. MNRAS 419, 573-607 (2012). doi:10.1111/j.1365-2966.2011.19721.x

M.V. Medvedev, A. Loeb, Generation of Magnetic Fields in the Relativistic Shock of Gamma-Ray Burst Sources. Astrophys. J. 526, 697-706 (1999). doi:10.1086/308038

P. Mészáros, M.J. Rees, Steep Slopes and Preferred Breaks in Gamma-Ray Burst Spectra: The Role of Photospheres and Comptonization. Astrophys. J. 530, 292-298 (2000). doi:10.1086/308371

P. Meszaros, P. Laguna, M.J. Rees, Gasdynamics of relativistically expanding gamma-ray burst sources - Kinematics, energetics, magnetic fields, and efficiency. Astrophys. J. 415, 181-190 (1993). doi: $10.1086 / 173154$

P. Mészáros, E. Ramirez-Ruiz, M.J. Rees, B. Zhang, X-Ray-rich Gamma-Ray Bursts, Photospheres, and Variability. Astrophys. J. 578, 812-817 (2002). doi:10.1086/342611

B.D. Metzger, E. Quataert, T.A. Thompson, Short-duration gamma-ray bursts with extended emission from protomagnetar spin-down. MNRAS 385, 1455-1460 (2008). doi:10.1111/j.1365-2966.2008.12923.x

B.D. Metzger, D. Giannios, T.A. Thompson, N. Bucciantini, E. Quataert, The protomagnetar model for gamma-ray bursts. MNRAS 413, 2031-2056 (2011). doi:10.1111/j.1365-2966.2011.18280.x

P. Mimica, M.A. Aloy, On the dynamic efficiency of internal shocks in magnetized relativistic outflows. MNRAS 401, 525-532 (2010). doi:10.1111/j.1365-2966.2009.15669.x

E. Molinari, et al., REM observations of GRB 060418 and GRB 060607A: the onset of the afterglow and the initial fireball Lorentz factor determination. Astron. Astrophys. 469, 13-16 (2007). doi:10.1051/0004-6361:20077388

C.G. Mundell, et al., Early Optical Polarization of a Gamma-Ray Burst Afterglow. Science 315, 1822 
(2007a). doi:10.1126/science.1138484

C.G. Mundell, et al., The Remarkable Afterglow of GRB 061007: Implications for Optical Flashes and GRB Fireballs. Astrophys. J. 660, 489-495 (2007b). doi:10.1086/512605

C.G. Mundell, et al., Highly polarized light from stable ordered magnetic fields in GRB 120308A. Nature 504, 119-121 (2013). doi:10.1038/nature12814

E. Nakar, Y. Oren, Polarization and Light-Curve Variability: The "Patchy-Shell" Model. Astrophys. J. Lett. 602, 97-100 (2004). doi:10.1086/382729

E. Nakar, R. Sari, Relativistic Shock Breakouts - A Variety of Gamma-Ray Flares: From Low-luminosity Gamma-Ray Bursts to Type Ia Supernovae. Astrophys. J. 747, 88 (2012). doi:10.1088/0004637X/747/2/88

E. Nakar, S. Ando, R. Sari, Klein-Nishina Effects on Optically Thin Synchrotron and Synchrotron SelfCompton Spectrum. Astrophys. J. 703, 675-691 (2009). doi:10.1088/0004-637X/703/1/675

E. Nakar, T. Piran, E. Waxman, Implications of the bold gamma-ray polarization of GRB 021206. J. Cosmo. Astro. Phys. 10, 5 (2003). doi:10.1088/1475-7516/2003/10/005

R. Narayan, P. Kumar, A. Tchekhovskoy, Constraints on cold magnetized shocks in gamma-ray bursts. MNRAS 416, 2193-2201 (2011). doi:10.1111/j.1365-2966.2011.19197.x

L. Nava, E. Nakar, T. Piran, Linear and circular polarization in GRB afterglows. ArXiv e-prints (2015)

L. Nava, G. Ghirlanda, G. Ghisellini, A. Celotti, Spectral properties of 438 GRBs detected by Fermi/GBM. Astron. Astrophys. 530, 21 (2011). doi:10.1051/0004-6361/201016270

J.A. Nousek, C. Kouveliotou, D. Grupe, K.L. Page, J. Granot, E. Ramirez-Ruiz, et al., Evidence for a Canonical Gamma-Ray Burst Afterglow Light Curve in the Swift XRT Data. Astrophys. J. 642, 389 400 (2006). doi:10.1086/500724

P.T. O'Brien, et al., The Early X-Ray Emission from GRBs. Astrophys. J. 647, 1213-1237 (2006). doi:10.1086/505457

B. Paczynski, Gamma-ray bursters at cosmological distances. Astrophys. J. Lett. 308, $43-46$ (1986). doi:10.1086/184740

A. Pe'er, Temporal Evolution of Thermal Emission from Relativistically Expanding Plasma. Astrophys. J. 682, 463-473 (2008). doi:10.1086/588136

A. Pe'er, P. Mészáros, M.J. Rees, The Observable Effects of a Photospheric Component on GRB and XRF Prompt Emission Spectrum. Astrophys. J. 642, 995-1003 (2006). doi:10.1086/501424

F. Peng, A. Königl, J. Granot, Two-Component Jet Models of Gamma-Ray Burst Sources. Astrophys. J. 626, 966-977 (2005). doi:10.1086/430045

D.A. Perley, et al., The Afterglow of GRB 130427A from 1 to $10^{16}$ GHz. Astrophys. J. 781, 37 (2014) doi:10.1088/0004-637X/781/1/37

T. Piran, Gamma-ray bursts and the fireball model. Physical Rept. 314, 575-667 (1999). doi:10.1016/S0370-1573(98)00127-6

T. Piran, The physics of gamma-ray bursts. Reviews of Modern Physics 76, 1143-1210 (2004). doi:10.1103/RevModPhys.76.1143

T. Piran, Magnetic Fields in Gamma-Ray Bursts: A Short Overview, in Magnetic Fields in the Universe: From Laboratory and Stars to Primordial Structures., ed. by E.M. de Gouveia dal Pino, G. Lugones, A. Lazarian American Institute of Physics Conference Series, vol. 784, 2005, pp. 164-174. doi:10.1063/1.2077181

T. Piran, E. Nakar, On the External Shock Synchrotron Model for Gamma-ray Bursts' GeV Emission. Astrophys. J. Lett. 718, 63-67 (2010). doi:10.1088/2041-8205/718/2/L63

T. Piran, R. Sari, Y.-C. Zou, Observational limits on inverse Compton processes in gamma-ray bursts MNRAS 393, 1107-1113 (2009). doi:10.1111/j.1365-2966.2008.14198.x

R. Preece, et al., The First Pulse of the Extremely Bright GRB 130427A: A Test Lab for Synchrotron Shocks. Science 343, 51-54 (2014). doi:10.1126/science.1242302

R.D. Preece, M.S. Briggs, R.S. Mallozzi, G.N. Pendleton, W.S. Paciesas, D.L. Band, The Synchrotron Shock Model Confronts a "Line of Death" in the BATSE Gamma-Ray Burst Data. Astrophys. J. Lett. 506, 23-26 (1998). doi:10.1086/311644

R.D. Preece, M.S. Briggs, R.S. Mallozzi, G.N. Pendleton, W.S. Paciesas, D.L. Band, The BATSE GammaRay Burst Spectral Catalog. I. High Time Resolution Spectroscopy of Bright Bursts Using High Energy Resolution Data. Astrophys. J. Supplements 126, 19-36 (2000). doi:10.1086/313289

W.H. Press, S.A. Teukolsky, W.T. Vetterling, B.P. Flannery, Numerical recipes in $C$. The art of scientific computing 1992

J.L. Racusin, S.V. Karpov, M. Sokolowski, J. Granot, et al., Broadband observations of the naked-eye $\gamma$-ray burst GRB080319B. Nature 455, 183-188 (2008). doi:10.1038/nature07270 
M.J. Rees, P. Meszaros, Unsteady outflow models for cosmological gamma-ray bursts. Astrophys. J. Lett. 430, 93-96 (1994). doi:10.1086/187446

M.J. Rees, P. Mészáros, Dissipative Photosphere Models of Gamma-Ray Bursts and X-Ray Flashes. Astrophys. J. 628, 847-852 (2005). doi:10.1086/430818

L. Rezzolla, P. Kumar, A Novel Paradigm for Short Gamma-Ray Bursts With Extended X-Ray Emission. Astrophys. J. 802, 95 (2015). doi:10.1088/0004-637X/802/2/95

S. Rosswog, Last Moments in the Life of a Compact Binary System: Gravitational Waves, Gamma-Ray Bursts and Magnetar Formation, in Revista Mexicana de Astronomia y Astrofisica, vol. 27. Revista Mexicana de Astronomia y Astrofisica, vol. 27, vol. 27, 2007, pp. 57-79

A. Rowlinson, et al., The unusual X-ray emission of the short Swift GRB 090515: evidence for the formation of a magnetar? MNRAS 409, 531-540 (2010). doi:10.1111/j.1365-2966.2010.17354.x

A. Rowlinson, P.T. O'Brien, B.D. Metzger, N.R. Tanvir, A.J. Levan, Signatures of magnetar central engines in short GRB light curves. MNRAS 430, 1061-1087 (2013). doi:10.1093/mnras/sts683

A. Rowlinson, B.P. Gompertz, M. Dainotti, P.T. O’Brien, R.A.M.J. Wijers, A.J. van der Horst, Constraining properties of GRB magnetar central engines using the observed plateau luminosity and duration correlation. MNRAS 443, 1779-1787 (2014). doi:10.1093/mnras/stu1277

R.E. Rutledge, D.B. Fox, Re-analysis of polarization in the $\gamma$-ray flux of GRB 021206. MNRAS 350 , 1288-1300 (2004). doi:10.1111/j.1365-2966.2004.07665.x

F. Ryde, et al., Identification and Properties of the Photospheric Emission in GRB090902B. Astrophys. J. Lett. 709, 172-177 (2010). doi:10.1088/2041-8205/709/2/L172

F. Ryde, et al., Observational evidence of dissipative photospheres in gamma-ray bursts. MNRAS 415 , 3693-3705 (2011). doi:10.1111/j.1365-2966.2011.18985.x

T. Sakamoto, et al., Global Characteristics of X-Ray Flashes and X-Ray-Rich Gamma-Ray Bursts Observed by HETE-2. Astrophys. J. 629, 311-327 (2005). doi:10.1086/431235

R. Sari, Linear Polarization and Proper Motion in the Afterglow of Beamed Gamma-Ray Bursts. Astrophys. J. Lett. 524, 43-46 (1999). doi:10.1086/312294

R. Sari, A.A. Esin, On the Synchrotron Self-Compton Emission from Relativistic Shocks and Its Implications for Gamma-Ray Burst Afterglows. Astrophys. J. 548, 787-799 (2001). doi:10.1086/319003

R. Sari, T. Piran, Hydrodynamic Timescales and Temporal Structure of Gamma-Ray Bursts. Astrophys. J. Lett. 455, 143 (1995). doi:10.1086/309835

R. Sari, T. Piran, Variability in Gamma-Ray Bursts: A Clue. Astrophys. J. 485, 270 (1997). doi: $10.1086 / 304428$

R. Sari, T. Piran, GRB 990123: The Optical Flash and the Fireball Model. Astrophys. J. Lett. 517, 109-112 (1999). doi:10.1086/312039

R. Sari, T. Piran, R. Narayan, Spectra and Light Curves of Gamma-Ray Burst Afterglows. Astrophys. J. Lett. 497, 17-20 (1998). doi:10.1086/311269

A. Shemi, T. Piran, The appearance of cosmic fireballs. Astrophys. J. Lett. 365, 55-58 (1990). doi: $10.1086 / 185887$

L. Sironi, A. Spitkovsky, Relativistic Reconnection: An Efficient Source of Non-thermal Particles. Astrophys. J. Lett. 783, 21 (2014). doi:10.1088/2041-8205/783/1/L21

L. Sironi, U. Keshet, M. Lemoine, Relativistic Shocks: Particle Acceleration and Magnetization. Space Science Reviews (2015). doi:10.1007/s11214-015-0102-2

A.M. Soderberg, et al., Relativistic ejecta from X-ray flash XRF 060218 and the rate of cosmic explosions Nature 442, 1014-1017 (2006). doi:10.1038/nature05087

A. Spitkovsky, Time-dependent Force-free Pulsar Magnetospheres: Axisymmetric and Oblique Rotators Astrophys. J. Lett. 648, 51-54 (2006). doi:10.1086/507518

H.C. Spruit, F. Daigne, G. Drenkhahn, Large scale magnetic fields and their dissipation in GRB fireballs. Astron. Astrophys. 369, 694-705 (2001). doi:10.1051/0004-6361:20010131

H.C. Spruit, T. Foglizzo, R. Stehle, Collimation of magnetically driven jets from accretion discs. MNRAS 288, 333-342 (1997)

I.A. Steele, C.G. Mundell, R.J. Smith, S. Kobayashi, C. Guidorzi, Ten per cent polarized optical emission from GRB090102. Nature 462, 767-769 (2009). doi:10.1038/nature08590

P.-H.T. Tam, Q.-W. Tang, S.-J. Hou, R.-Y. Liu, X.-Y. Wang, Discovery of an Extra Hard Spectral Component in the High-energy Afterglow Emission of GRB 130427A. Astrophys. J. Lett. 771, 13 (2013). doi:10.1088/2041-8205/771/1/L13

A. Tchekhovskoy, R. Narayan, J.C. McKinney, Magnetohydrodynamic simulations of gammaray burst jets: Beyond the progenitor star. New Astronomy 15, 749-754 (2010) doi:10.1016/j.newast.2010.03.001 
C. Thompson, A Model of Gamma-Ray Bursts. MNRAS 270, 480 (1994)

E. Troja, et al., Swift Observations of GRB 070110: An Extraordinary X-Ray Afterglow Powered by the Central Engine. Astrophys. J. 665, 599-607 (2007). doi:10.1086/519450

Z.L. Uhm, A.M. Beloborodov, On the Mechanism of Gamma-Ray Burst Afterglows. Astrophys. J. Lett. 665, 93-96 (2007). doi:10.1086/519837

Z.L. Uhm, B. Zhang, Fast-cooling synchrotron radiation in a decaying magnetic field and $\gamma$-ray burst emission mechanism. Nature Physics 10, 351-356 (2014). doi:10.1038/nphys2932

Z.L. Uhm, B. Zhang, R. Hascoët, F. Daigne, R. Mochkovitch, I.H. Park, Dynamics and Afterglow Light Curves of Gamma-Ray Burst Blast Waves with a Long-lived Reverse Shock. Astrophys. J. 761, 147 (2012). doi:10.1088/0004-637X/761/2/147

V.V. Usov, Millisecond pulsars with extremely strong magnetic fields as a cosmological source of gammaray bursts. Nature 357, 472-474 (1992). doi:10.1038/357472a0

W.T. Vestrand, et al., The Bright Optical Flash and Afterglow from the Gamma-Ray Burst GRB 130427A. Science 343, 38-41 (2014). doi:10.1126/science.1242316

M. Vietri, L. Stella, A Gamma-Ray Burst Model with Small Baryon Contamination. Astrophys. J. Lett. 507, 45-48 (1998). doi:10.1086/311674

M. Vietri, L. Stella, Supranova Events from Spun-up Neutron Stars: An Explosion in Search of an Observation. Astrophys. J. Lett. 527, 43-46 (1999). doi:10.1086/312386

F.J. Virgili, E.-W. Liang, B. Zhang, Low-luminosity gamma-ray bursts as a distinct GRB population: a firmer case from multiple criteria constraints. MNRAS 392, 91-103 (2009). doi:10.1111/j.13652966.2008.14063.x

I. Vurm, A.M. Beloborodov, J. Poutanen, Gamma-Ray Bursts from Magnetized Collisionally Heated Jets. Astrophys. J. 738, 77 (2011). doi:10.1088/0004-637X/738/1/77

I. Vurm, Y. Lyubarsky, T. Piran, On Thermalization in Gamma-Ray Burst Jets and the Peak Energies of Photospheric Spectra. Astrophys. J. 764, 143 (2013). doi:10.1088/0004-637X/764/2/143

X.-Y. Wang, Z. Li, Z.-G. Dai, P. Mészáros, GRB 080916C: On the Radiation Origin of the Prompt Emission from keV/MeV TO GeV. Astrophys. J. Lett. 698, 98-102 (2009). doi:10.1088/0004-637X/698/2/L98

K. Wiersema, et al., Circular polarization in the optical afterglow of GRB 121024A. Nature 509, 201-204 (2014). doi:10.1038/nature13237

C. Wigger, W. Hajdas, K. Arzner, M. Güdel, A. Zehnder, Gamma-Ray Burst Polarization: Limits from RHESSI Measurements. Astrophys. J. 613, 1088-1100 (2004). doi:10.1086/423163

R. Willingale, F. Genet, J. Granot, P.T. O'Brien, The spectral-temporal properties of the prompt pulses and rapid decay phase of gamma-ray bursts. MNRAS 403, 1296-1316 (2010). doi:10.1111/j.13652966.2009.16187.x

S.E. Woosley, Gamma-ray bursts from stellar mass accretion disks around black holes. Astrophys. J. 405, 273-277 (1993). doi:10.1086/172359

S.E. Woosley, J.S. Bloom, The Supernova Gamma-Ray Burst Connection. ARAA 44, 507-556 (2006). doi:10.1146/annurev.astro.43.072103.150558

D. Yonetoku, et al., Detection of Gamma-Ray Polarization in Prompt Emission of GRB 100826A. Astrophys. J. Lett. 743, 30 (2011). doi:10.1088/2041-8205/743/2/L30

D. Yonetoku, et al., Magnetic Structures in Gamma-Ray Burst Jets Probed by Gamma-Ray Polarization. Astrophys. J. Lett. 758, 1 (2012). doi:10.1088/2041-8205/758/1/L1

B. Zhang, et al., Physical Processes Shaping Gamma-Ray Burst X-Ray Afterglow Light Curves: Theoretical Implications from the Swift X-Ray Telescope Observations. Astrophys. J. 642, 354-370 (2006). doi:10.1086/500723

B. Zhang, H. Yan, The Internal-collision-induced Magnetic Reconnection and Turbulence (ICMART) Model of Gamma-ray Bursts. Astrophys. J. 726, 90 (2011). doi:10.1088/0004-637X/726/2/90

B. Zhang, B. Zhang, Gamma-Ray Burst Prompt Emission Light Curves and Power Density Spectra in the ICMART Model. Astrophys. J. 782, 92 (2014). doi:10.1088/0004-637X/782/2/92

W. Zhang, S.E. Woosley, A.I. MacFadyen, Relativistic Jets in Collapsars. Astrophys. J. 586, 356-371 (2003). doi:10.1086/367609

X. Zhao, Z. Li, X. Liu, B.-b. Zhang, J. Bai, P. Mészáros, Gamma-Ray Burst Spectrum with Decaying Magnetic Field. Astrophys. J. 780, 12 (2014). doi:10.1088/0004-637X/780/1/12 